\title{
Debating Lapita: Distribution, chronology, society and subsistence
}

\author{
Stuart Bedford, Matthew Spriggs, David V. Burley, Christophe Sand, \\ Peter Sheppard and Glenn R. Summerhayes
}

Lapita has been a focus for archaeologists for generations. Initially inspired by the scattered reporting in the early twentieth century of highly decorated sherds (Meyer 1909; McKern 1929; Piroutet 1917), its increasing significance in terms of the human settlement of the Pacific began to build in the 1960s. The Lapita culture has been most clearly defined by its distinctive dentatestamped decorated pottery and the design system represented on it and on further incised pots. It is defined earliest in the Bismarck Archipelago to the east of the large island of New Guinea, at some time in the centuries preceding $3000 \mathrm{cal}$. BP. At around that date the Lapita culture spread out from its Bismarck Archipelago 'homeland' to beyond previously inhabited regions of Near Oceania to establish the first human colonies in the western part of Remote Oceania, the present-day south-east Solomons, Vanuatu, New Caledonia, Fiji, Tonga, Samoa and Wallis and Futuna. At about the same time there was a push to the south-west out from the Bismarcks along the south coast of New Guinea, perhaps even as far as the Torres Straits (McNiven et al. 2006).

The broad overlapping themes of this volume, Lapita distribution and chronology, society and subsistence, relate to research questions that have long been debated in relation to Lapita. It is a substantial volume with 23 chapters, reflecting the increasing breadth and focus on different aspects of Lapita that have developed over several decades.

The distribution and chronology theme, as addressed in Chapters 2 to 9, connects to questions of its geographical spread, site location within that extent and its origins. The eastern and southern boundaries appear to be well established, having remained unchanged since the 1970s: Samoa the furthest east and New Caledonia the most southerly. However, it seems very unlikely the north-western boundaries of Lapita are yet fixed, with earlier and simpler dentate-stamping having been found in Island Southeast Asia (ISEA) (e.g. Hung et al. 2011 for Luzon). How these ISEA pottery traditions relate to dentate-stamped pottery in Micronesia and Lapita in the Bismarcks remains unresolved. Some have argued that the pottery follows a trail from ISEA to Micronesia and then on to the Bismarcks (Carson 2018; Carson et al. 2013). Clark and Winter (Chapter 2) address this question head-on with a detailed comparison of motif forms and designs from the two regions. In an expansion of the boundary, recent serendipitous discoveries and predictive modelling of the $3000 \mathrm{cal}$. BP south Papuan coastline have now extended the Lapita range westwards right up to the Fly River (Skelly et al. 2014). David et al. (Chapter 3) discuss one of these sites, Moiapu at Caution Bay near Port Moresby, and how it fits into the regional context and sequence, focusing particularly on its place at the end of Lapita. These discoveries on the south Papuan coast, along with later pottery from the Torres Strait (McNiven et al. 2006) 
and on Lizard Island on Australia's Great Barrier Reef (Tochilin et al. 2012), suggest that the western and south-western boundaries of Lapita are not yet fixed. The implications of this are discussed by Lilley (Chapter 5), who suggests that coastal Aboriginal Australians were most likely much more connected to Lapita expansion than previously imagined, both linking to it and also involved in facilitating its extension.

The theme of distribution also connects with questions of site location and spread within the Lapita boundary. Sites are overwhelmingly found in coastal locales but can be found on large islands through to small islets, on back beaches or in intertidal situations. The evidence for the latter appears to be largely restricted to Near Oceania where Lapita populations were entering already populated islands (but see Burley 2016, Nunn and Heorake 2009 for suggested Tongan and Fijian examples). Two chapters address these different locales. Summerhayes et al. (Chapter 4) focus on the Near Oceanian site of Kamgot, an Early Lapita site in the Anir Group off southern New Ireland, once located in an intertidal zone. They outline the archaeological evidence and discuss the economic advantages of establishing a settlement in an intertidal situation. Burley et al. (Chapter 8) discuss an Early Lapita site in Fiji, on the small island of Kavewa in Vanua Levu. It is strategically positioned on a passage through the reef, a location that may have been chosen by founder colonies to facilitate exploration and settlement of the Vanua Levu mainland.

While the geographic distribution of Lapita dentate-stamped sites has been extended, there remain some persistent gaps within its current extension, namely the central Solomons, Samoa and American Samoa. There remains an ongoing debate whether these are real or perceived. In some cases, the geomorphological and post-depositional complexities make the search and discovery of Lapita sites extremely difficult. A lack of focused research is also a factor in some areas. This might be partly the case in the Vitiaz Strait where Lapita has been found only on the Siassi Islands to date, but it seems most likely that it may have been more widespread due to the strategic location between the Bismarcks and the New Guinea mainland. Gaffney et al. (Chapter 6) address this question in relation to Arop Island where recent fieldwork has been carried out. They present a range of evidence that points to a Lapita presence on that island. Sheppard (Chapter 7) revisits the Lapita gap in the main Solomons, arguing that earlier contentions for a leap-frog scenario of Early Lapita settlement remain robust. He adds new data that further support this scenario.

Lapita chronology is continually being addressed, and many of the chapters here touch on this aspect. Long gone are the days when Lapita was seen to continue in some areas for more than a thousand years, or that there was a pause in the Bismarcks for 400 years before people moved further east. Much more refined use of radiometric methods, the incorporation of $\Delta \mathrm{R}$ offsets for marine shell samples, the identification of charcoal to species, or the specific targeting of short-lived samples such as nutshell have led to much more robust chronological delineation. The use of uranium-thorium dating (U/Th) on coral artefacts has also provided unprecedented chronological definition in the case of Tonga. What the dating of Lapita sites in Remote Oceania currently supports is a scenario where populations 'exploded out of the Bismarcks, and in a radiocarbon instant, occupied most of its ultimate range' (Sheppard 2010:107). The time period for the production of dentate-stamped pottery has also been generally reduced across the same region, with its demise now appearing to be no later than 2700-2500 cal. BP. The further delineation of the chronology for Lapita in Near Oceania remains to be completed. A major review of dates for that region is the focus of Chapter 9 (Specht and Gosden). The authors radically trim down the numbers of acceptable dates for the region under a chronometric hygiene exercise and also suggest a likely starting date for Lapita in the Bismarcks could be around 3250-3150 cal. BP. Kirch, in his closing commentary (Chapter 23), states that his own soon-to-be-published re-dating of Lapita in the Mussau group will present a similar time range for that part of the Lapita 'homeland' area as well. 
Chapters 10 to 17 are situated under the broad category of Lapita society with a particular focus on aspects of the design system and its symbolism (Chapters 12 to 16). A range of comparative techniques and statistical analyses are employed, all of which tease out various aspects of the design system and its application. Results of these analyses also contribute to discussions relating to connectivity, colonisation strategies and chronology. Sand et al. (Chapter 10) revisit the eponymous Site 13, Lapita, following extensive rescue archaeology excavations carried out there in 2015. New dates and new data provide a much fuller picture of the history of the site's occupation over several hundred years. The results highlight the spatial complexity of the site, which is a cautionary tale for Lapita research in general, as summaries of sites and whole islands can be based on only a few test pits that may represent 5 per cent or less of a site overall. Moving east to Vanuatu, Bedford illustrates and discusses Lapita pottery, both vessel form and designs, recovered from the small islands of north-east Malakula (Chapter 11). While the sites clearly date to the colonising phase in this part of Vanuatu, the pottery displays very distinctive regional variation. This leads to questions both of ultimate origins and about the rates of change in any given ceramic sequence. The topics of the influence and origins of the Lapita design system are taken up by Ambrose in Chapter 12. Ambrose, who first illustrated Lapita sherds in a 1959 publication (Golson 1959), up-ends the argument that Lapita motifs influenced design systems on other media. He argues, rather, that plaiting and basketry were more likely to have been dominant and fundamental influences on Oceanic art, including the decoration of Lapita pottery (Chapter 12). Lapita 'faces' have long been argued as being an essential component of Lapita iconography. Spriggs, who first established this (Spriggs 1990), brings a totally new perspective to the debate (Chapter 13). Here he argues that what once were seen as faces are more likely to represent heads and masks, headdresses and skullcaps.

The chapter of LeBlanc et al. (Chapter 14) is the first of three that look at Lapita motifs and design structure in fine detail. LeBlanc et al. shift away from the more traditional element-motif approach by taking a structural approach to analysing the Lapita design system, targeting design density, layout and organisation. Their focus, using this approach, is on assessing how cohesive the Eastern Lapita Province is in terms of ceramic design. Chiu (Chapter 15) provides an update of her ongoing research into Lapita motifs, presenting data on motif similarity among 50 Lapita sites, highlighting social connections that may be traced through the distribution of motifs. Along similar lines of inquiry to those of Chiu, Noury (Chapter 16), who argues that there are two primary distinct sets of Lapita designs or group designs, uses them to track movement across Remote Oceania. Rather than focusing on Lapita design, Leclerc (Chapter 17) discusses the results of the chemical characterisation of Lapita and Post-Lapita ceramics. He argues that the shift from the compositional variability found in Lapita ceramics to a restricted compositional range found in Post-Lapita ceramics is an indication of significant societal change.

Chapters 18 to 21 focus on aspects of Lapita subsistence. The chapters highlight the radically different environments that were encountered by Lapita populations and the range of food sources that were available and exploited. Summerhayes et al. (Chapter 18) provide a detailed study of midden remains from the Early Lapita site of Kamgot and its distinctive intertidal location. Lebot and Sam (Chapter 19) review the availability of indigenous plants in Vanuatu that could have been consumed by Lapita colonists. They suggest that a whole range of plant food sources could potentially have been utilised on first arrival, weakening any argument that settlement in this part of Remote Oceania was hindered by a paucity of locally available flora. Ono et al. (Chapter 20) present a review of Lapita fishing, providing a case study from the small island of Uripiv adjacent to Malakula in Vanuatu and comparing it with all other published Lapita sites. Fish bone remains, fishhooks and capture methods are discussed. Hawkins and Worthy (Chapter 21) review avian extinctions across the Lapita distribution, highlighting the evidence for radical impact that humans had on a range of naïve and vulnerable species. The importance of taphonomic influence in assessing site preservation and thus impact is also highlighted. 
Chapter 22 (Shing and Willie) moves beyond the strictly academic focus on Lapita to show how it has been accepted or not in wider educational and community awareness programs. The authors discuss how the long-running archaeology awareness programs in Vanuatu, coordinated by the Vanuatu Cultural Centre, have developed in different and sometimes unexpected directions.

Patrick Kirch first encountered Lapita sites in the field in 1971 on a visit to the Reef Islands in the south-east Solomons, and first excavated them on the island of Futuna in Western Polynesia in 1974. In the final chapter (Chapter 23), he reflects on four decades of Lapita research and what the priorities might be for the future.

\section{Inventory of Lapita sites}

The first tally of Lapita sites was compiled by Green in 1979. It included a map (1979: Figure 2.2), discussion of site stratigraphy and excavated areas, pottery recovered, a mere 24 radiocarbon dates (1979: Table 2.10) and relevant references (1979:49-57). There were 19 localities and around 60 sites. Kirch and Hunt (1988) followed 10 years later with similar detail and reached a figure of 79 sites. Another 10 years after that, Kirch estimated that there were around 100 sites, and described in some detail 32 of those where information was easily accessible (1997:263276). He noted that for the remaining two-thirds of the inventory 'very little is known indeed' (Kirch 1997:263).

In 2001 the number of sites had increased to 184, and a full list and accompanying data and references were tabulated (Anderson et al. 2001). The last full tally, although not a full listing, was in 2007 where the number of sites had increased to 229 (Bedford and Sand 2007:8-9). As part of the overall preparation of this volume, we have compiled an updated inventory of all sites where dentate-stamped pottery has been found in the Western Pacific. We follow the historical pattern of such inventories in that we include sites where a dentate-stamped component of decoration is included in the ceramics recovered. There are two exceptions included where Lapita sites are indicated through other evidence. They are a site at Lamap, Malakula, Vanuatu, where a single piece of Talasea obsidian was collected on the surface and the site of Pouebo in New Caledonia where the very distinctive geology of this region has been identified in Lapita sherds found from other sites in New Caledonia (Chiu et al. 2016). Table 1.1 follows earlier formats and includes national site register codes if available, site name, a general description of locality, site extent, contents, the ceramic series in terms of the localised sequence, age in calendrical years BP (gleaned from relevant radiocarbon dates or comparison of design motifs) and key references.

Since the last tally of sites in 2007, we have added 64 sites to the inventory, providing now an overall total of 293 Lapita sites across its distribution. While this appears to be a substantial increase it is not all newly discovered sites. The new total has come about both through the addition of new sites (49) that have been identified since 2007, but also the addition of a number of old sites (15) from the Bismarcks (Kombe and Fissoa) and the south-east Solomons (Taumako and Reefs-Santa Cruz) that managed to slip through previous tallies. One site that was previously incorrectly listed was also removed (Loloma, New Georgia). Totals for each region are now as follows: 19 for New Guinea, 88 for the Bismarcks, 28 for the Solomons, 30 for Vanuatu, 38 for New Caledonia, 51 for Fiji, 34 for Tonga, one for Samoa, three for Wallis (East Uvea) and one for Futuna. We do not include sites in ISEA or the Marianas where dentate-stamped pottery is known or designs in incised or other techniques display complex 'Lapita-like' motifs whose exact relation to Western Pacific Lapita have yet to be established (see Anggraeni et al. 2014; Aoyagi et al. 1993; Azis et al. 2018; Bellwood and Koon 1989; Carson 2014; Chia 2003; Hung 2008; Lape 2000). Similarly, the early pottery sites in the Torres Strait and the undated finds on Lizard Island on Queensland's Great Barrier Reef are not included because of the non-specific 
nature of pottery found so far at these sites (see Lilley, Chapter 5, for a discussion of these sites). The new sites that have been identified since 2007 include 17 in New Guinea, two in the Bismarcks, three in the Solomons, five in Vanuatu, three in New Caledonia, seven in Fiji and 11 in Tonga. In most regions, the increase of new sites has been slowly incremental, as per the focus of archaeological research in any particular area, which is to be expected. The most striking addition is the extension of the distribution of Lapita further west along the south coast of New Guinea (David et al. 2011; Skelly et al. 2014). However, in arriving at a total of 16 sites for Caution Bay we have followed the definition used by the excavators, where an archaeological 'site' was defined as a location of cultural materials $15 \mathrm{~m}$ or more from its closest neighbour. This recording criteria tends to inflate occupational settlement site numbers in relation to other areas of the Lapita distribution.

Table 1.1. Currently known Lapita sites (293): Location, locale type, extent, content, ceramic series, age and references.

\begin{tabular}{|c|c|c|c|c|c|c|c|}
\hline Code & Location name & $\begin{array}{l}\text { Locale } \\
\text { type }\end{array}$ & $\begin{array}{l}\text { Extent } \\
(\mathrm{sq} \mathrm{m})\end{array}$ & Content & $\begin{array}{l}\text { Ceramic } \\
\text { series }\end{array}$ & Age BP & References \\
\hline \multicolumn{8}{|c|}{ North New Guinea } \\
\hline \multicolumn{8}{|c|}{ Saudaun Province } \\
\hline No code & Aitape & unknown & - & $\begin{array}{l}\text { surface } \\
\text { pottery }\end{array}$ & ?Middle & - & Swadling et al. 1988 \\
\hline \multicolumn{8}{|l|}{ Sepik } \\
\hline RNJ & $\begin{array}{l}\text { Tubungbale, } \\
\text { Ali Island }\end{array}$ & coastal flat & - & $\begin{array}{l}\text { surface } \\
\text { pottery }\end{array}$ & ?Late & - & \begin{tabular}{|l} 
Terrell and Welsch \\
1997 \\
\end{tabular} \\
\hline \multicolumn{8}{|c|}{ South New Guinea } \\
\hline \multicolumn{8}{|c|}{ Gulf Province } \\
\hline 0JS & $\begin{array}{l}\text { Hopo, Vailala } \\
\text { River }\end{array}$ & $\begin{array}{l}\text { inland } \\
\text { ancient } \\
\text { dune }\end{array}$ & - & pottery & Late & $2668-2615$ & Skelly et al. 2014 \\
\hline \multicolumn{8}{|c|}{ Caution Bay } \\
\hline ABEN & Bogi 1 & $\begin{array}{l}\text { coastal } \\
\text { midden }\end{array}$ & 1500 & full range & $\begin{array}{l}\text { Middle } \\
\text { to Late }\end{array}$ & $2900-2250$ & $\begin{array}{l}\text { McNiven et al. 2011; } \\
\text { David et al. } 2011\end{array}$ \\
\hline ABHA & Tanamu 1 & $\begin{array}{l}\text { coastal } \\
\text { midden }\end{array}$ & 260 & $\begin{array}{l}\text { pottery and } \\
\text { obsidian }\end{array}$ & $\begin{array}{l}\text { Middle } \\
\text { to Late }\end{array}$ & $2900-2860$ & $\begin{array}{l}\text { David et al. 2011; } \\
\text { Mialanes et al. } 2016\end{array}$ \\
\hline ABHD & Tanamu 3 & $\begin{array}{l}\text { coastal } \\
\text { midden }\end{array}$ & - & $\begin{array}{l}\text { pottery and } \\
\text { obsidian }\end{array}$ & Late & $2750-2350$ & \\
\hline AAYN & Moiapu 1 & $\begin{array}{l}\text { inland } \\
\text { low hill }\end{array}$ & - & $\begin{array}{l}\text { pottery and } \\
\text { obsidian }\end{array}$ & Late & $2551-2470$ & $\begin{array}{l}\text { David et al. 2011; } \\
\text { Mialanes et al. } 2016\end{array}$ \\
\hline AAYL & Moiapu 2 & $\begin{array}{l}\text { Inland } \\
\text { low hill }\end{array}$ & - & $\begin{array}{l}\text { pottery and } \\
\text { obsidian }\end{array}$ & Late & $2700-2250$ & Mialanes et al. 2016 \\
\hline AAZD & Moiapu 3 & $\begin{array}{l}\text { inland } \\
\text { low hill }\end{array}$ & - & $\begin{array}{l}\text { pottery and } \\
\text { obsidian }\end{array}$ & Late & $2630-2410$ & $\begin{array}{l}\text { David et al. this } \\
\text { volume }\end{array}$ \\
\hline AAWA & Nese 1 & $\begin{array}{l}\text { inland } \\
\text { low hill }\end{array}$ & - & $\begin{array}{l}\text { pottery and } \\
\text { obsidian }\end{array}$ & Late & $2750-2550$ & $\begin{array}{l}\text { McNiven et al. 2012a: } \\
\text { Figure 2; Mialanes et } \\
\text { al. } 2016\end{array}$ \\
\hline ABA0 & Edubu 1 & $\begin{array}{l}\text { inland } \\
\text { low hill }\end{array}$ & 300 & full range & Late & $2650-2350$ & McNiven et al. 2012b \\
\hline ABAN & Edubu 2 & $\begin{array}{l}\text { inland } \\
\text { low hill }\end{array}$ & - & pottery & Late & $2850-2150$ & Mialanes et al. 2016 \\
\hline ABKL & ML 18 & $\begin{array}{l}\text { coastal } \\
\text { midden }\end{array}$ & - & $\begin{array}{l}\text { pottery and } \\
\text { obsidian }\end{array}$ & Late & $2850-2650$ & Mialanes et al. 2016 \\
\hline AAUJ & JA21 & inland & - & $\begin{array}{l}\text { pottery and } \\
\text { obsidian }\end{array}$ & Late & $2800-2550$ & Mialanes et al. 2016 \\
\hline
\end{tabular}




\begin{tabular}{|c|c|c|c|c|c|c|c|}
\hline Code & Location name & $\begin{array}{l}\text { Locale } \\
\text { type }\end{array}$ & $\begin{array}{l}\text { Extent } \\
\text { (sq m) }\end{array}$ & Content & $\begin{array}{l}\text { Ceramic } \\
\text { series }\end{array}$ & Age BP & References \\
\hline AAIT & MLA14 & $\begin{array}{l}\text { inland } \\
\text { low hill }\end{array}$ & - & pottery & Late & $2750-2500$ & Mialanes et al. 2016 \\
\hline AAVM & Ataga 1 & $\begin{array}{l}\text { inland } \\
\text { low hill }\end{array}$ & - & $\begin{array}{l}\text { pottery and } \\
\text { obsidian }\end{array}$ & Late & $2650-2450$ & $\begin{array}{l}\text { McNiven et al. 2011; } \\
\text { Mialanes et al. } 2016\end{array}$ \\
\hline- & JD17 & $\begin{array}{l}\text { coastal } \\
\text { midden }\end{array}$ & - & pottery & Late & - & $\begin{array}{l}\text { McNiven et al. 2011: } \\
\text { Figure 5n }\end{array}$ \\
\hline- & JD10 & $\begin{array}{l}\text { coastal } \\
\text { midden }\end{array}$ & - & pottery & Late & - & McNiven et al. 2011 \\
\hline ABIV & JD14 & $\begin{array}{l}\text { coastal } \\
\text { midden }\end{array}$ & - & pottery & Late & - & $\begin{array}{l}\text { McNiven et al. 2011; } \\
\text { McNiven et al. 2012a: } \\
\text { Figure } 2\end{array}$ \\
\hline \multicolumn{8}{|c|}{ Morobe Province } \\
\hline KLK & $\begin{array}{l}\text { Tuam Island, } \\
\text { Siassi }\end{array}$ & coastal flat & 2000 & pottery & $\begin{array}{l}\text { Middle } \\
\text { to Late }\end{array}$ & $3150-2750$ & Lilley 2002 \\
\hline \multicolumn{8}{|c|}{ West New Britain } \\
\hline \multicolumn{8}{|c|}{ Arawes } \\
\hline FNY & Paligmete & $\begin{array}{l}\text { coastal } \\
\text { midden }\end{array}$ & 18000 & full range & Early & - & Summerhayes 2000a \\
\hline FNZ & Winguru & $\begin{array}{l}\text { coastal } \\
\text { midden }\end{array}$ & 18000 & full range & Late & - & Summerhayes 2000a \\
\hline $\mathrm{FOH}$ & Magekur & $\begin{array}{l}\text { coastal } \\
\text { midden }\end{array}$ & 10000 & full range & \begin{tabular}{|l|} 
Early \\
to Middle
\end{tabular} & $3240-2750$ & Summerhayes 2000a \\
\hline F0J & Apalo & $\begin{array}{l}\text { coastal } \\
\text { midden }\end{array}$ & 12000 & full range & \begin{tabular}{|l|} 
Early \\
to Late
\end{tabular} & $3200-2520$ & Summerhayes 2000a \\
\hline FOL & Amalut & $\begin{array}{l}\text { coastal } \\
\text { midden }\end{array}$ & 3000 & full range & $\begin{array}{l}\text { Middle } \\
\text { to Late }\end{array}$ & $2770-2360$ & $\begin{array}{l}\text { Specht and Gosden } \\
1997\end{array}$ \\
\hline No code & Maklo & $\begin{array}{l}\text { coastal } \\
\text { midden }\end{array}$ & - & $\begin{array}{l}\text { pottery and } \\
\text { obsidian }\end{array}$ & - & - & Swadling 1992 \\
\hline FOR & Maklo & $\begin{array}{l}\text { coastal } \\
\text { midden }\end{array}$ & - & $\begin{array}{l}\text { pottery and } \\
\text { obsidian }\end{array}$ & - & - & Swadling 1992 \\
\hline FOF & Lolmo & $\begin{array}{l}\text { offshore } \\
\text { island cave }\end{array}$ & 55 & full range & Late & - & Gosden et al. 1994 \\
\hline & Agussak & $\begin{array}{l}\text { offshore } \\
\text { island }\end{array}$ & 500 & $\begin{array}{l}\text { pottery and } \\
\text { obsidian }\end{array}$ & - & - & Swadling 1992 \\
\hline \multicolumn{8}{|l|}{ Kandrian } \\
\hline \multicolumn{8}{|c|}{ Kandrian area } \\
\hline FLF & Alanglongromo & rock shelter & 30 & $\begin{array}{l}\text { pottery and } \\
\text { obsidian }\end{array}$ & Middle & $3060-2750$ & Summerhayes 2000a \\
\hline FLK & Aringilo & open site & - & pottery & - & - & Specht 1991a \\
\hline FLX & Ngaikwo & open site & - & pottery & - & - & Specht 1991a \\
\hline FYA & Narangpun & open site & - & pottery & - & - & Specht et al. 1992 \\
\hline FFT & Langpun & \begin{tabular}{|l} 
coastal \\
midden \\
\end{tabular} & - & pottery & - & - & Specht et al. 1981 \\
\hline FFS & Auгагu0 & $\begin{array}{l}\text { coastal } \\
\text { midden }\end{array}$ & - & pottery & $\begin{array}{l}\text { Middle } \\
\text { to Late }\end{array}$ & - & Summerhayes 2000a \\
\hline FNT & Kreslo & $\begin{array}{l}\text { reef } \\
\text { platform }\end{array}$ & 2500 & pottery & $\begin{array}{l}\text { Middle } \\
\text { to Late }\end{array}$ & - & Specht 1991b \\
\hline \multicolumn{8}{|c|}{ Talasea area } \\
\hline $\mathrm{FCN} / \mathrm{FCO}$ & Point Mondu & beach & - & $\begin{array}{l}\text { pottery and } \\
\text { obsidian }\end{array}$ & - & - & $\begin{array}{l}\text { Specht and Torrence } \\
\text { 2007a }\end{array}$ \\
\hline FCR/FCS & $\begin{array}{l}\text { Lagenda } \\
\text { plantation }\end{array}$ & beach & - & $\begin{array}{l}\text { pottery } \\
\text { obsidian }\end{array}$ & Early & - & Specht et al. 1988 \\
\hline
\end{tabular}




\begin{tabular}{|c|c|c|c|c|c|c|c|}
\hline Code & Location name & $\begin{array}{l}\text { Locale } \\
\text { type }\end{array}$ & $\begin{array}{l}\text { Extent } \\
\text { (sq m) }\end{array}$ & Content & $\begin{array}{l}\text { Ceramic } \\
\text { series }\end{array}$ & Age BP & References \\
\hline FCT & Lagenda Island & beach & - & pottery & - & - & $\begin{array}{l}\text { Specht and Torrence } \\
\text { 2007a }\end{array}$ \\
\hline FDK & Nariri Beach & beach & - & $\begin{array}{l}\text { pottery and } \\
\text { obsidian }\end{array}$ & - & - & $\begin{array}{l}\text { Specht and Torrence } \\
\text { 2007a }\end{array}$ \\
\hline $\mathrm{FCH}$ & Nabodu beach & beach & - & pot sherd & - & - & $\begin{array}{l}\text { Specht and Torrence } \\
\text { 2007a }\end{array}$ \\
\hline FRJ & Valahia & beach & - & $\begin{array}{l}\text { pottery and } \\
\text { obsidian }\end{array}$ & - & - & $\begin{array}{l}\text { Specht and Torrence } \\
2007 \text { a }\end{array}$ \\
\hline FRI & Walindi & inland spur & - & $\begin{array}{l}\text { pottery and } \\
\text { obsidian }\end{array}$ & - & - & $\begin{array}{l}\text { Specht and Torrence } \\
\text { 2007a }\end{array}$ \\
\hline \multicolumn{8}{|c|}{ Garua Harbour } \\
\hline FEA & Boduna & islet & 6000 & $\begin{array}{l}\text { pottery and } \\
\text { obsidian }\end{array}$ & $\begin{array}{l}\text { Early to } \\
\text { Middle }\end{array}$ & $2950-2720$ & $\begin{array}{l}\text { Specht and } \\
\text { Summerhayes } 2007\end{array}$ \\
\hline FEM & Garala Island & $\begin{array}{l}\text { offshore } \\
\text { island }\end{array}$ & - & $\begin{array}{l}\text { pottery and } \\
\text { obsidian }\end{array}$ & - & - & $\begin{array}{l}\text { Specht and Torrence } \\
\text { 2007a }\end{array}$ \\
\hline FQD & $\begin{array}{l}\text { Langu, Binnen } \\
\text { Island }\end{array}$ & $\begin{array}{l}\text { offshore } \\
\text { island }\end{array}$ & - & $\begin{array}{l}\text { pottery and } \\
\text { obsidian }\end{array}$ & - & - & $\begin{array}{l}\text { Specht and Torrence } \\
\text { 2007a }\end{array}$ \\
\hline \multicolumn{8}{|l|}{ Garua Island } \\
\hline FSZ & Scoria pit & coastal hill & 1600 & $\begin{array}{l}\text { pottery and } \\
\text { obsidian }\end{array}$ & $\begin{array}{l}\text { Middle } \\
\text { to Late }\end{array}$ & $2800-2000$ & Summerhayes 2000a \\
\hline FAO & unknown & coastal hill & 1600 & $\begin{array}{l}\text { pottery and } \\
\text { obsidian }\end{array}$ & $\begin{array}{l}\text { Middle } \\
\text { to Late }\end{array}$ & - & $\begin{array}{l}\text { Specht and Torrence } \\
\text { 2007a }\end{array}$ \\
\hline FQY & Golas Gully & coastal hill & - & $\begin{array}{l}\text { pottery and } \\
\text { obsidian }\end{array}$ & - & - & $\begin{array}{l}\text { Specht and Torrence } \\
\text { 2007a }\end{array}$ \\
\hline FAS & unknown & $\begin{array}{l}\text { coastal } \\
\text { stream }\end{array}$ & - & sherd & - & - & $\begin{array}{l}\text { Specht and Torrence } \\
\text { 2007a }\end{array}$ \\
\hline FEK & Mt America & mudflats & - & sherd & Early & - & $\begin{array}{l}\text { Specht and Torrence } \\
\text { 2007a }\end{array}$ \\
\hline FEL & unknown & coastal hill & - & $\begin{array}{l}\text { pottery and } \\
\text { obsidian }\end{array}$ & \begin{tabular}{|l} 
Early \\
to Late
\end{tabular} & - & $\begin{array}{l}\text { Specht and Torrence } \\
\text { 2007a }\end{array}$ \\
\hline FXO & unknown & coastal hill & - & $\begin{array}{l}\text { pottery and } \\
\text { obsidian }\end{array}$ & Late & - & $\begin{array}{l}\text { Torrence and } \\
\text { Stevenson } 2000\end{array}$ \\
\hline FYS & unknown & beach & - & pottery & - & - & $\begin{array}{l}\text { Specht and Torrence } \\
\text { 2007a }\end{array}$ \\
\hline $\mathrm{FCY}$ & unknown & beach & - & $\begin{array}{l}\text { pottery and } \\
\text { obsidian }\end{array}$ & Late & - & $\begin{array}{l}\text { Specht and Torrence } \\
\text { 2007a }\end{array}$ \\
\hline FAAN/D5-7 & unknown & $\begin{array}{l}\text { coastal } \\
\text { plain }\end{array}$ & - & $\begin{array}{l}\text { pottery and } \\
\text { obsidian }\end{array}$ & $\begin{array}{l}\text { Early } \\
\text { to Late }\end{array}$ & 2700 & $\begin{array}{l}\text { Specht and Torrence } \\
\text { 2007a }\end{array}$ \\
\hline FAAJ & unknown & $\begin{array}{l}\text { coastal } \\
\text { plain }\end{array}$ & - & $\begin{array}{l}\text { pottery and } \\
\text { obsidian }\end{array}$ & - & - & $\begin{array}{l}\text { Specht and Torrence } \\
\text { 2007a }\end{array}$ \\
\hline FAAQ & unknown & inland hill & - & $\begin{array}{l}\text { pottery and } \\
\text { obsidian }\end{array}$ & - & - & $\begin{array}{l}\text { Specht and Torrence } \\
2007 \text { a }\end{array}$ \\
\hline \multicolumn{8}{|c|}{ Willaumez Peninsula } \\
\hline FAAH & \begin{tabular}{|l} 
Numundo \\
plantation
\end{tabular} & coastal hill & - & $\begin{array}{l}\text { pottery and } \\
\text { obsidian }\end{array}$ & - & $3200-2960$ & Torrence et al. 1999 \\
\hline FABH & $\begin{array}{l}\text { Numundo } \\
\text { plantation }\end{array}$ & inland hill & - & $\begin{array}{l}\text { pottery and } \\
\text { obsidian }\end{array}$ & Late & - & Torrence et al. 1999 \\
\hline FABN & Garu plantation & inland hill & - & $\begin{array}{l}\text { surface } \\
\text { pottery }\end{array}$ & - & - & Torrence et al. 1999 \\
\hline FACU & unknown & $\begin{array}{l}\text { hill on } \\
\text { divide }\end{array}$ & - & $\begin{array}{l}\text { surface } \\
\text { pottery }\end{array}$ & - & - & $\begin{array}{l}\text { Specht and Torrence } \\
2007 b\end{array}$ \\
\hline
\end{tabular}




\begin{tabular}{|c|c|c|c|c|c|c|c|}
\hline Code & Location name & $\begin{array}{l}\text { Locale } \\
\text { type }\end{array}$ & $\begin{array}{l}\text { Extent } \\
\text { (sq m) }\end{array}$ & Content & $\begin{array}{l}\text { Ceramic } \\
\text { series }\end{array}$ & Age BP & References \\
\hline FACZ & $\begin{array}{l}\text { Foothill of } \\
\text { Mt Krummel }\end{array}$ & $\begin{array}{l}\text { inland } \\
\text { foothill }\end{array}$ & - & $\begin{array}{l}\text { surface } \\
\text { pottery }\end{array}$ & - & - & $\begin{array}{l}\text { Specht and Torrence } \\
2007 \mathrm{~b}\end{array}$ \\
\hline FACR & $\begin{array}{l}\text { Whiteman range } \\
\text { foothills }\end{array}$ & low spurs & - & $\begin{array}{l}\text { surface } \\
\text { pottery }\end{array}$ & Late & 2800 & $\begin{array}{l}\text { Specht and Torrence } \\
\text { 2007b }\end{array}$ \\
\hline \multicolumn{8}{|c|}{ Kombe Coast } \\
\hline $\mathrm{FCL}$ & Poi Mission & $\begin{array}{l}\text { coastal } \\
\text { midden }\end{array}$ & - & full range & - & $3000-2700$ & Lilley 1991 \\
\hline FPA & Kautaga Island & $\begin{array}{l}\text { coastal } \\
\text { midden }\end{array}$ & - & full range & - & $3000-2700$ & Lilley 1991 \\
\hline FPB & Kou Island & midden & - & pottery & - & - & Lilley 1991 \\
\hline FPN & Rudiger Point & midden & - & pottery & - & - & Lilley 1991 \\
\hline FPR & Poi Island & $\begin{array}{l}\text { coastal } \\
\text { midden }\end{array}$ & - & pottery & - & - & Lilley 1991 \\
\hline FPE & Kalapia Island & midden & - & pottery & - & - & Lilley 1991 \\
\hline FPF & Kalapia Island & midden & - & pottery & - & - & Lilley 1991 \\
\hline \multicolumn{8}{|c|}{ East New Britain } \\
\hline \multicolumn{8}{|c|}{ Duke of Yorks } \\
\hline \multicolumn{8}{|c|}{ Duke of York Island } \\
\hline SDN & Piuka & $\begin{array}{l}\text { coastal } \\
\text { midden }\end{array}$ & - & $\begin{array}{l}\text { pottery and } \\
\text { obsidian }\end{array}$ & - & - & White and Harris 1997 \\
\hline SDK & Urkuk & $\begin{array}{l}\text { coastal } \\
\text { midden }\end{array}$ & - & pottery & - & - & White 2007 \\
\hline SDP & Kabilomo & $\begin{array}{l}\text { coastal } \\
\text { midden }\end{array}$ & - & $\begin{array}{l}\text { pottery and } \\
\text { obsidian }\end{array}$ & - & - & White 2007 \\
\hline SES/SET & Nakukur 1 and 2 & $\begin{array}{l}\text { coastal } \\
\text { midden }\end{array}$ & $75000 ?$ & $\begin{array}{l}\text { pottery and } \\
\text { obsidian }\end{array}$ & - & - & White 2007 \\
\hline \multicolumn{8}{|l|}{ Mioko } \\
\hline SDQ & Mioko Island & $\begin{array}{l}\text { coastal } \\
\text { midden }\end{array}$ & - & pottery & - & - & White 2007 \\
\hline SFB/SFA & Palpal village & $\begin{array}{l}\text { coastal } \\
\text { midden }\end{array}$ & 30000 & pottery & - & - & White 2007 \\
\hline \multicolumn{8}{|l|}{ Kabakon } \\
\hline SEE & Kabakon Island & $\begin{array}{l}\text { coastal } \\
\text { midden }\end{array}$ & 8100 & full range & Early & 3090 & White 2007 \\
\hline \multicolumn{8}{|l|}{ Utuan } \\
\hline SFF & unknown & $\begin{array}{l}\text { coastal } \\
\text { midden }\end{array}$ & - & $\begin{array}{l}\text { pottery and } \\
\text { obsidian }\end{array}$ & - & - & White 2007 \\
\hline \multicolumn{8}{|l|}{ Kerawara } \\
\hline SEF & unknown & $\begin{array}{l}\text { coastal } \\
\text { midden }\end{array}$ & - & $\begin{array}{l}\text { pottery and } \\
\text { obsidian }\end{array}$ & - & - & White 2007 \\
\hline \multicolumn{8}{|l|}{ Makada } \\
\hline SEO & unknown & $\begin{array}{l}\text { coastal } \\
\text { midden }\end{array}$ & - & $\begin{array}{l}\text { pottery and } \\
\text { obsidian }\end{array}$ & - & - & White 2007 \\
\hline SEP & Uraputput Point & $\begin{array}{l}\text { coastal } \\
\text { midden }\end{array}$ & - & $\begin{array}{l}\text { pottery and } \\
\text { obsidian }\end{array}$ & - & $>2780$ & White 2007 \\
\hline \multicolumn{8}{|l|}{ Watom } \\
\hline \multicolumn{8}{|c|}{ Reber Mission and Rakival } \\
\hline SAC & Kainapirina & $\begin{array}{l}\text { coastal } \\
\text { midden }\end{array}$ & - & full range & $\begin{array}{l}\text { Middle to } \\
\text { Late }\end{array}$ & $2200-2000$ & Anson et al. 2005 \\
\hline
\end{tabular}




\begin{tabular}{|c|c|c|c|c|c|c|c|}
\hline Code & Location name & $\begin{array}{l}\text { Locale } \\
\text { type }\end{array}$ & $\begin{array}{l}\text { Extent } \\
(\mathrm{sq} \mathrm{m})\end{array}$ & Content & $\begin{array}{l}\text { Ceramic } \\
\text { series }\end{array}$ & Age BP & References \\
\hline SDI & Vunavaung & $\begin{array}{l}\text { coastal } \\
\text { midden }\end{array}$ & - & full range & $\begin{array}{l}\text { Middle } \\
\text { to Late }\end{array}$ & $2800-1800$ & Anson et al. 2005 \\
\hline SAD & Maravot & $\begin{array}{l}\text { coastal } \\
\text { midden }\end{array}$ & - & full range & $\begin{array}{l}\text { Middle } \\
\text { to Late }\end{array}$ & $2300-1900$ & Anson et al. 2005 \\
\hline SAU & Vunailau & $\begin{array}{l}\text { coastal } \\
\text { hill/cliff }\end{array}$ & - & $\begin{array}{l}\text { surface } \\
\text { pottery, } \\
\text { obsidian }\end{array}$ & - & - & $\begin{array}{l}\text { Specht 1968; Anson } \\
\text { pers. comm. } 2017\end{array}$ \\
\hline \multicolumn{8}{|c|}{ Pomio- Jacquinot Bay } \\
\hline No code & Liton River & river & - & pottery & Late? & - & $\begin{array}{l}\text { Leavesley and Sarar } \\
2013\end{array}$ \\
\hline \multicolumn{8}{|c|}{ New Ireland } \\
\hline \multicolumn{8}{|c|}{ New Ireland mainland } \\
\hline EFY & Lemau & $\begin{array}{l}\text { coastal } \\
\text { midden }\end{array}$ & - & $\begin{array}{l}\text { pottery, } \\
\text { obsidian, } \\
\text { stone }\end{array}$ & - & - & White 1992 \\
\hline EAA & Lossu & $\begin{array}{l}\text { coastal } \\
\text { midden }\end{array}$ & - & pottery & - & - & $\begin{array}{l}\text { White and Downie } \\
1980\end{array}$ \\
\hline ELS/ELT & Lasigi & $\begin{array}{l}\text { coastal } \\
\text { midden }\end{array}$ & - & pottery & Late & $2980-2690$ & Golson 1992 \\
\hline No code & Bagail Kavieng & $\begin{array}{l}\text { coastal } \\
\text { midden }\end{array}$ & - & $\begin{array}{l}\text { pottery, } \\
\text { obsidian }\end{array}$ & Late? & - & $\begin{array}{l}\text { Summerhayes and } \\
\text { Leavesley pers. comm. }\end{array}$ \\
\hline ENX & Fissoa & $\begin{array}{l}\text { coastal } \\
\text { midden }\end{array}$ & - & pottery & Late? & - & $\begin{array}{l}\text { White and Murray- } \\
\text { Wallace } 1996\end{array}$ \\
\hline \multicolumn{8}{|c|}{ Lambon Island } \\
\hline EPE & Lambon Island & unknown & - & pottery & - & - & White 1996 \\
\hline \multicolumn{8}{|c|}{ Anir Islands } \\
\hline EAQ & Malekolon & $\begin{array}{l}\text { coastal } \\
\text { midden }\end{array}$ & 10000 & $\begin{array}{l}\text { pottery, } \\
\text { obsidian, } \\
\text { vol. stone }\end{array}$ & Middle & $2900-2300$ & Summerhayes 2000b \\
\hline ERA & Kamgot & $\begin{array}{l}\text { coastal } \\
\text { midden }\end{array}$ & 24000 & full range & Early & $3200-2900$ & Summerhayes 2000b \\
\hline ERB & Mission & $\begin{array}{l}\text { coastal } \\
\text { midden }\end{array}$ & - & $\begin{array}{l}\text { pottery, } \\
\text { obsidian, } \\
\text { vol. stone }\end{array}$ & Middle & $2900-2300$ & Summerhayes 2000b \\
\hline No code & Naliu & $\begin{array}{l}\text { coastal } \\
\text { midden }\end{array}$ & - & $\begin{array}{l}\text { pottery and } \\
\text { obsidian }\end{array}$ & $\begin{array}{l}\text { Middle/ } \\
\text { Late }\end{array}$ & $2900-2300$ & Summerhayes 2000b \\
\hline ERC & Balbalankin & $\begin{array}{l}\text { coastal } \\
\text { midden }\end{array}$ & 15000 & full range & Middle & $2900-2300$ & Summerhayes 2000b \\
\hline \multicolumn{8}{|l|}{ Mussau } \\
\hline $\mathrm{ECA}$ & Talepakemalai & $\begin{array}{l}\text { coastal } \\
\text { midden }\end{array}$ & 72500 & full range & Early & $3300-2770$ & Kirch 1997; Kirch 2001 \\
\hline $\mathrm{ECB}$ & Etakosarai & $\begin{array}{l}\text { coastal } \\
\text { midden }\end{array}$ & 2700 & full range & Early? & $3500-3300$ & Kirch et al. 1991 \\
\hline EHB & Etapakengaroasa & $\begin{array}{l}\text { coastal } \\
\text { midden }\end{array}$ & 1150 & full range & - & $3500-2400$ & Kirch et al. 1991 \\
\hline EKQ & Epakapaka & rock shelter & 90 & full range & Middle? & $3100-2800$ & Kirch et al. 1991 \\
\hline \multicolumn{8}{|c|}{ Emira Island } \\
\hline EQS & Tamuarawai & coastal flat & 22500 & full range & Early & $3300-3000$ & $\begin{array}{l}\text { Summerhayes et al. } \\
2010\end{array}$ \\
\hline
\end{tabular}




\begin{tabular}{|c|c|c|c|c|c|c|c|}
\hline Code & Location name & $\begin{array}{l}\text { Locale } \\
\text { type }\end{array}$ & $\begin{array}{l}\text { Extent } \\
\text { (sq m) }\end{array}$ & Content & $\begin{array}{l}\text { Ceramic } \\
\text { series }\end{array}$ & Age BP & References \\
\hline \multicolumn{8}{|c|}{ Tanga Island } \\
\hline ETM & Angkitkita & coastal flat & - & $\begin{array}{l}\text { pottery and } \\
\text { lithics }\end{array}$ & Late & 2750 & $\begin{array}{l}\text { Garling 2003; } \\
\text { Cath-Garling } 2017\end{array}$ \\
\hline \multicolumn{8}{|c|}{ Admiralties } \\
\hline \multicolumn{8}{|c|}{ Manus } \\
\hline GDN & Kohin Cave & cave & - & pottery & - & $3900-2450$ & Kennedy 1981 \\
\hline GLT & Mouk & coastal flat & - & pottery & - & - & $\begin{array}{l}\text { McEldowney and } \\
\text { Ballard } 1991\end{array}$ \\
\hline GFR & $\begin{array}{l}\text { Paemasa } \\
\text { (Baluan) }\end{array}$ & coastal flat & - & pottery & - & - & $\begin{array}{l}\text { McEldowney and } \\
\text { Ballard } 1991 \\
\end{array}$ \\
\hline \multicolumn{8}{|c|}{ SOLOMONS } \\
\hline \multicolumn{8}{|c|}{ North Solomons } \\
\hline \multicolumn{8}{|c|}{ Nissan } \\
\hline DFF & Lebang Halika & rock shelter & - & full range & Early & - & Spriggs 1991 \\
\hline $\mathrm{DGD} / 2$ & Unknown & rock shelter & 40 & full range & Early & $>2730$ & Spriggs 1991 \\
\hline DES & Tarmon & reef flat & 5110 & $\begin{array}{l}\text { pottery and } \\
\text { adzes }\end{array}$ & Late & - & Spriggs 1991 \\
\hline \multicolumn{8}{|c|}{ Buka and Sohano } \\
\hline DJQ & Kessa & reef flat & 10000 & $\begin{array}{l}\text { pottery, } \\
\text { obsidian, } \\
\text { vol. stone, } \\
\text { adzes }\end{array}$ & Middle & - & Wickler 2001 \\
\hline DAF & Sohano & reef flat & 39000 & $\begin{array}{l}\text { pottery, } \\
\text { obsidian, } \\
\text { vol. stone, } \\
\text { adzes }\end{array}$ & Middle & - & Wickler 2001 \\
\hline DAA & Sohano & rock shelter & - & $\begin{array}{l}\text { pottery and } \\
\text { obsidian }\end{array}$ & Middle & - & Wickler 2001 \\
\hline DKC & $\begin{array}{l}\text { Sohano Primary } \\
\text { School }\end{array}$ & rock shelter & 100 & pottery & Middle & - & Wickler 2001 \\
\hline \multicolumn{8}{|c|}{ New Georgia } \\
\hline \multicolumn{8}{|c|}{ Roviana Lagoon } \\
\hline Site 97 & $\begin{array}{l}\text { Honiavasa, } \\
\text { Honiavasa Island }\end{array}$ & intertidal & 1800 & pottery & Late & - & Felgate 2001, 2003 \\
\hline Site 96 & $\begin{array}{l}\text { Nusa Roviana } \\
\text { (Zoroka) }\end{array}$ & intertidal & 1750 & pottery & Late & - & Felgate 2001, 2003 \\
\hline \multicolumn{8}{|c|}{ Kolombangara } \\
\hline No code & $\begin{array}{l}\text { Poitete, } \\
\text { Kolombangara } \\
\text { Island } \\
\end{array}$ & intertidal & - & $\begin{array}{l}\text { surface } \\
\text { sherds }\end{array}$ & Late & - & $\begin{array}{l}\text { Summerhayes and } \\
\text { Scales } 2005\end{array}$ \\
\hline \multicolumn{8}{|c|}{ SE SOLOMONS } \\
\hline \multicolumn{8}{|c|}{ Taumako } \\
\hline SE-DF-19 & $\begin{array}{l}\text { Te Ana Tavatava, } \\
\text { Lakao Island }\end{array}$ & rock shelter & - & full range & Late & $2950-2350$ & $\begin{array}{l}\text { Leach and Davidson } \\
2008\end{array}$ \\
\hline \multicolumn{8}{|c|}{ Reef-Santa Cruz } \\
\hline SE-RF-2 & $\begin{array}{l}\text { Nenumbo, Te } \\
\text { Motu Taibä, } \\
\text { Ngaua, Reef } \\
\text { Islands }\end{array}$ & $\begin{array}{l}\text { coastal } \\
\text { midden }\end{array}$ & 1000 & full range & Middle & $3185-2639$ & $\begin{array}{l}\text { Green 1976; } \\
\text { Sheppard et al. } 2015\end{array}$ \\
\hline
\end{tabular}




\begin{tabular}{|c|c|c|c|c|c|c|c|}
\hline Code & Location name & $\begin{array}{l}\text { Locale } \\
\text { type }\end{array}$ & $\begin{array}{l}\text { Extent } \\
(\mathrm{sq} \mathrm{m})\end{array}$ & Content & $\begin{array}{l}\text { Ceramic } \\
\text { series }\end{array}$ & Age BP & References \\
\hline SE-RF-4 & $\begin{array}{l}\text { Te Motu Taibä, } \\
\text { Ngaua, Reef } \\
\text { Islands }\end{array}$ & $\begin{array}{l}\text { coastal } \\
\text { beach }\end{array}$ & - & $\begin{array}{l}\text { surface } \\
\text { scatter } \\
\text { pottery }\end{array}$ & - & - & Green 1979 \\
\hline SE-RF-5 & $\begin{array}{l}\text { Laki, Te Motu } \\
\text { Taibä, Ngaua, } \\
\text { Reef Islands }\end{array}$ & surface & - & $\begin{array}{l}\text { surface } \\
\text { pottery }\end{array}$ & - & - & Doherty 2007 \\
\hline SE-RF-6 & $\begin{array}{l}\text { Ngamanie, } \\
\text { Lomlom, Reef } \\
\text { Islands }\end{array}$ & $\begin{array}{l}\text { coastal } \\
\text { midden }\end{array}$ & 2400 & $\begin{array}{l}\text { pottery and } \\
\text { midden, } \\
\text { obsidian, } \\
\text { chert }\end{array}$ & Middle & $2910-2470$ & $\begin{array}{l}\text { Green 1976; Green } \\
\text { and Jones } 2007\end{array}$ \\
\hline SE-RF-7 & $\begin{array}{l}\text { Te Motu Taibä, } \\
\text { Ngaua, Reef } \\
\text { Islands }\end{array}$ & $\begin{array}{l}\text { small } \\
\text { surface } \\
\text { scatter }\end{array}$ & - & pottery & - & - & Green 1979 \\
\hline SE-RF-8 & $\begin{array}{l}\text { Fenualoa, Reef } \\
\text { Islands }\end{array}$ & $\begin{array}{l}\text { surface } \\
\text { scatter }\end{array}$ & 2400 & pottery & - & - & Green 1979 \\
\hline SE-SZ-8 & $\begin{array}{l}\text { Nanggu, Nendö } \\
\text { [traditional } \\
\text { name for Santa } \\
\text { Cruz] Santa Cruz }\end{array}$ & $\begin{array}{l}\text { coastal } \\
\text { midden }\end{array}$ & 14000 & $\begin{array}{l}\text { pottery and } \\
\text { midden, } \\
\text { obsidian, } \\
\text { chert } \\
\end{array}$ & Middle & $2920-2729$ & $\begin{array}{l}\text { Green 1976; Green } \\
\text { et al. 2008; Sheppard } \\
\text { et al. } 2015\end{array}$ \\
\hline SE-SZ-10 & $\begin{array}{l}\text { Tömotu Noi } \\
\text { Island, Santa } \\
\text { Cruz }\end{array}$ & $\begin{array}{l}\text { coastal } \\
\text { midden }\end{array}$ & - & $\begin{array}{l}\text { surface } \\
\text { collected } \\
\text { mainly } \\
\text { plain, } 1 \\
\text { piece of } \\
\text { obsidian }\end{array}$ & - & - & $\begin{array}{l}\text { Green 1976; Green } \\
\text { et al. } 2008\end{array}$ \\
\hline SE-SZ-23 & $\begin{array}{l}\text { Malu, Tömotu } \\
\text { Neo Island, } \\
\text { Santa Cruz } \\
\end{array}$ & $\begin{array}{l}\text { coastal } \\
\text { midden }\end{array}$ & 3000 & $\begin{array}{l}\text { pottery, } \\
\text { chert, } \\
\text { obsidian }\end{array}$ & Middle & - & $\begin{array}{l}\text { McCoy and Cleghorn } \\
1988\end{array}$ \\
\hline SE-SZ-33 & $\begin{array}{l}\text { Mdailu, Santa } \\
\text { Cruz }\end{array}$ & $\begin{array}{l}\text { coastal } \\
\text { midden }\end{array}$ & - & pottery & - & - & $\begin{array}{l}\text { McCoy and Cleghorn } \\
1988\end{array}$ \\
\hline SE-SZ-42 & $\begin{array}{l}\text { Luenemba River, } \\
\text { Santa Cruz }\end{array}$ & $\begin{array}{l}\text { ceramics in } \\
\text { stream cut }\end{array}$ & - & pottery & - & - & Green et al. 2008 \\
\hline SE-SZ-45 & $\begin{array}{l}\text { Bianga Mepala, } \\
\text { Wia Island, } \\
\text { Santa Cruz }\end{array}$ & $\begin{array}{l}\text { coastal } \\
\text { midden }\end{array}$ & 10000 & $\begin{array}{l}\text { pottery, } \\
\text { obsidian, } \\
\text { adze }\end{array}$ & Middle & - & $\begin{array}{l}\text { McCoy and Cleghorn } \\
1988\end{array}$ \\
\hline SE-SZ-50 & $\begin{array}{l}\text { Bonati, Tömotu } \\
\text { Noi, Santa Cruz }\end{array}$ & surface & - & pottery & Middle? & - & Green et al. 2008 \\
\hline \multicolumn{8}{|l|}{ Vanikoro } \\
\hline No code & Ngae & - & - & pottery & - & - & $\begin{array}{l}\text { Noury and Galipaud } \\
2011\end{array}$ \\
\hline No code & Milu & - & - & pottery & - & - & $\begin{array}{l}\text { Noury and Galipaud } \\
2011\end{array}$ \\
\hline No code & Lavaka & - & - & pottery & - & - & $\begin{array}{l}\text { Noury and Galipaud } \\
2011\end{array}$ \\
\hline \multicolumn{8}{|l|}{ Tikopia } \\
\hline TK 4 & Kiki & $\begin{array}{l}\text { coastal } \\
\text { midden }\end{array}$ & 4500 & full range & Late & $2900-2800$ & $\begin{array}{l}\text { Kirch and Yen 1982; } \\
\text { Kirch and Swift } 2017\end{array}$ \\
\hline \multicolumn{8}{|l|}{ VANUATU } \\
\hline \multicolumn{8}{|c|}{ Mota Lava, Banks Islands } \\
\hline No code & Nerenugman & back beach & 3000 & full range & $\begin{array}{l}\text { Middle } \\
\text { to Late }\end{array}$ & - & $\begin{array}{l}\text { Bedford and Spriggs } \\
2014\end{array}$ \\
\hline
\end{tabular}




\begin{tabular}{|c|c|c|c|c|c|c|c|}
\hline Code & Location name & $\begin{array}{l}\text { Locale } \\
\text { type }\end{array}$ & $\begin{array}{l}\text { Extent } \\
(\mathrm{sq} \mathrm{m})\end{array}$ & Content & $\begin{array}{l}\text { Ceramic } \\
\text { series }\end{array}$ & Age BP & References \\
\hline \multicolumn{8}{|l|}{ Santo } \\
\hline No code & $\begin{array}{l}\text { Big Bay } \\
\text { Matantas }\end{array}$ & back beach & 3500 & \begin{tabular}{|l} 
pottery and \\
obsidian
\end{tabular} & Late & $2900-2800$ & $\begin{array}{l}\text { Bedford and Spriggs } \\
2008\end{array}$ \\
\hline No code & Port Olry & back beach & 3000 & $\begin{array}{l}\text { pottery and } \\
\text { obsidian }\end{array}$ & $\begin{array}{l}\text { Middle } \\
\text { to Late }\end{array}$ & $2900-2800$ & $\begin{array}{l}\text { Bedford and Spriggs } \\
\text { 2008; Bedford } \\
\text { fieldnotes }\end{array}$ \\
\hline No code & Shograon & back beach & - & pottery & $\begin{array}{l}\text { Middle } \\
\text { to Late }\end{array}$ & 2500 & $\begin{array}{l}\text { Galipaud 2010; Noury } \\
\text { and Galipaud } 2011\end{array}$ \\
\hline \multicolumn{8}{|l|}{ Aоге } \\
\hline No code & Makué & back beach & - & full range & $\begin{array}{l}\text { Early to } \\
\text { Late }\end{array}$ & $3150-2950$ & $\begin{array}{l}\text { Galipaud and Swete } \\
\text { Kelly 2007; Galipaud } \\
\text { et al. } 2014\end{array}$ \\
\hline No code & west coast & back beach & - & $\begin{array}{l}\text { surface } \\
\text { pottery }\end{array}$ & - & - & Galipaud 2001 \\
\hline No code & SDA Mission & back beach & - & $\begin{array}{l}\text { surface } \\
\text { pottery }\end{array}$ & - & - & $\begin{array}{l}\text { Galipaud 2001; Noury } \\
\text { and Galipaud } 2011\end{array}$ \\
\hline \multicolumn{8}{|l|}{ Tutuba } \\
\hline No code & east coast & back beach & - & $\begin{array}{l}\text { surface } \\
\text { pottery }\end{array}$ & - & - & Galipaud 2001 \\
\hline No code & south-east coast & back beach & - & $\begin{array}{l}\text { surface } \\
\text { pottery }\end{array}$ & - & - & Galipaud 2001 \\
\hline \multicolumn{8}{|l|}{ Mavea } \\
\hline No code & north-east coast & coastal flat & - & pottery & - & - & $\begin{array}{l}\text { Galipaud and Vienne } \\
2005\end{array}$ \\
\hline No code & east coast & coastal flat & - & pottery & - & - & $\begin{array}{l}\text { Galipaud and Vienne } \\
\text { 2005; Bedford and } \\
\text { Galipaud } 2010\end{array}$ \\
\hline \multicolumn{8}{|l|}{ Malo } \\
\hline MA 8-20 & Batuni-urunga & coastal flat & $>3000$ & full range & Middle & $3000-2800$ & Hedrick n.d. \\
\hline MA 8-38 & Avunatari & coastal flat & $>3000$ & full range & Middle & $3000-2800$ & Galipaud 1998 \\
\hline MA 8-39 & Naone & coastal flat & $>3000$ & full range & Middle & $3000-2800$ & Hedrick n.d. \\
\hline MA 8-40 & Atanoasao & coastal flat & - & full range & Middle & $3000-2800$ & $\begin{array}{l}\text { Galipaud 1998; Bedford } \\
\text { and Galipaud } 2010 \\
\end{array}$ \\
\hline No code & Avnambulu & coastal flat & - & pottery & - & & Hedrick 1971 \\
\hline No code & Alawara & coastal flat & - & & - & & Hedrick 1971 \\
\hline \multicolumn{8}{|l|}{ Malakula } \\
\hline No code & Malua Bay & back beach & $10-100$ & full range & Late & $2800-2600$ & Bedford 2006a \\
\hline No code & Uripiv Island & back beach & 2000 & full range & Late & $2850-2600$ & $\begin{array}{l}\text { Bedford 2003; Horrocks } \\
\text { and Bedford 2005; } \\
\text { Bedford et al. 2011; } \\
\text { Kinaston et al. } 2014\end{array}$ \\
\hline No code & Wala Island & back beach & 1000 & full range & Late & $2800-2600$ & Bedford 2003 \\
\hline No code & Atchin Island & back beach & 2000 & \begin{tabular}{|l|} 
full range \\
\end{tabular} & Late & $2800-2600$ & \begin{tabular}{|l|} 
Bedford 2003 \\
\end{tabular} \\
\hline No code & Vao Island & back beach & 3000 & full range & $\begin{array}{l}\text { Middle } \\
\text { to Late }\end{array}$ & $3000-2600$ & $\begin{array}{l}\text { Bedford 2003, 2006b; } \\
\text { Bedford et al. } 2011\end{array}$ \\
\hline No code & Lamap & surface & - & \begin{tabular}{|l} 
Talasea \\
obsidian \\
\end{tabular} & - & - & Bedford fieldnotes \\
\hline No code & Port Stanley & surface & - & $\begin{array}{l}\text { dentate } \\
\text { sherd }\end{array}$ & Middle & & Bedford fieldnotes \\
\hline
\end{tabular}




\begin{tabular}{|c|c|c|c|c|c|c|c|}
\hline Code & Location name & $\begin{array}{l}\text { Locale } \\
\text { type }\end{array}$ & $\begin{array}{l}\text { Extent } \\
(\mathrm{sq} \mathrm{m})\end{array}$ & Content & $\begin{array}{l}\text { Ceramic } \\
\text { series }\end{array}$ & Age BP & References \\
\hline \multicolumn{8}{|l|}{ Efate } \\
\hline No code & Teouma & $\begin{array}{l}\text { back beach } \\
\text { promontory }\end{array}$ & 2000 & full range & $\begin{array}{l}\text { Early } \\
\text { to Late }\end{array}$ & $3000-2800$ & $\begin{array}{l}\text { Bedford et al. 2010; } \\
\text { Petchey et al. 2014, } \\
2015\end{array}$ \\
\hline No code & Teouma west & surface & - & $\begin{array}{l}\text { dentate } \\
\text { sherd }\end{array}$ & Early & $3000-2800$ & $\begin{array}{l}\text { Shing and Willie this } \\
\text { volume }\end{array}$ \\
\hline No code & Erueti & back beach & $1000-2000$ & full range & $\begin{array}{l}\text { Middle } \\
\text { to Late }\end{array}$ & $3000-2800$ & $\begin{array}{l}\text { Garanger 1972; Bedford } \\
\text { and Spriggs } 2014\end{array}$ \\
\hline \multicolumn{8}{|l|}{ Erromango } \\
\hline No code & Ifo & coastal flat & $1000-2000$ & full range & $\begin{array}{l}\text { Middle } \\
\text { to Late }\end{array}$ & $3000-2800$ & Bedford 2006a \\
\hline No code & Ponamla & back beach & $100-500$ & full range & Late & 2800 & $\begin{array}{l}\text { Bedford et al. 1998; } \\
\text { Bedford 2006a }\end{array}$ \\
\hline \multicolumn{8}{|l|}{ Aneityum } \\
\hline No code & Anelguhuat & back beach & 3000 & full range & Late & 2800 & Bedford et al. 2016 \\
\hline \multicolumn{8}{|c|}{ NEW CALEDONIA } \\
\hline \multicolumn{8}{|c|}{ North coast } \\
\hline NKM001 & Boirra & coastal flat & 10000 & full range & $\begin{array}{l}\text { Early } \\
\text { to Late }\end{array}$ & $3000-2750$ & Galipaud 1988 \\
\hline NAR098 & Arama & coastal flat & - & pottery & ? Late & - & Galipaud 1988 \\
\hline NPL001 & Pam & mangrove & - & pottery & ? Late & - & Sand et al. 2001 \\
\hline \multicolumn{8}{|l|}{ West coast } \\
\hline WK0027 & Oundjo & coastal flat & - & pottery & ?Late & - & Baret et al. 2000 \\
\hline WK0013 & Lapita & coastal flat & 500 & full range & $\begin{array}{l}\text { Early } \\
\text { to Late }\end{array}$ & - & $\begin{array}{l}\text { Gifford and Shutler } \\
1956\end{array}$ \\
\hline WK0013A & Lapita & coastal flat & 20000 & full range & $\begin{array}{l}\text { Early } \\
\text { to Late }\end{array}$ & $3000-2750$ & $\begin{array}{l}\text { Sand 1998a; Sand et al. } \\
\text { this volume }\end{array}$ \\
\hline WK0013B & Lapita & coastal flat & 250 & full range & $\begin{array}{l}\text { Early } \\
\text { to Late }\end{array}$ & $3000-2750$ & Sand 1998a \\
\hline WK0014 & Podtanean & coastal flat & - & pot sherd & ?Late & - & $\begin{array}{l}\text { Gifford and Shutler } \\
1956\end{array}$ \\
\hline WK0028 & Koniene & coastal flat & - & pottery & ?Late & - & Sand 1996 \\
\hline WK0141 & Podtanean & coastal flat & - & pottery & ?Late & - & Galipaud 1988 \\
\hline WNP003 & Franco & $\begin{array}{l}\text { coastal } \\
\text { sand }\end{array}$ & - & pot sherd & ?Late & - & Galipaud 1988 \\
\hline WNP038 & Pindai & $\begin{array}{l}\text { coastal } \\
\text { sand }\end{array}$ & - & pottery & ?Late & - & Sand 1996 \\
\hline WBR001 & Nessadiou & $\begin{array}{l}\text { coastal } \\
\text { sand }\end{array}$ & - & full range & $\begin{array}{l}\text { Early } \\
\text { to Late }\end{array}$ & $3000-2750$ & Sand 1996 \\
\hline WBR009 & île Verte & $\begin{array}{l}\text { coastal } \\
\text { dune }\end{array}$ & - & pot sherd & ?Late & - & $\begin{array}{l}\text { Frimigacci and Siorat } \\
1988\end{array}$ \\
\hline WBR006 & Temroc & coastal flat & - & pottery & ?Late & - & Frimigacci 1975 \\
\hline WPT055 & Naïa & coastal flat & - & pottery & ?Late & - & Smart n.d. \\
\hline WPT055 & Naïa & coastal flat & - & pottery & $\begin{array}{l}\text { ?Early } \\
\text { to Late }\end{array}$ & - & Frimigacci 1975 \\
\hline WPT148 & Ongoué & coastal flat & 10000 & pottery & $\begin{array}{l}\text { ?Early } \\
\text { to Late }\end{array}$ & - & Sand 1994 \\
\hline V8 & Vavouto & coastal flat & 10000 & full range & $\begin{array}{l}\text { Early } \\
\text { to Late }\end{array}$ & $2900-2750$ & Sand 2010 \\
\hline GD 2006-042 & Deva & coastal flat & no estimate & pottery & ?Late & - & Barp et al. 2006 \\
\hline
\end{tabular}




\begin{tabular}{|c|c|c|c|c|c|c|c|}
\hline Code & Location name & $\begin{array}{l}\text { Locale } \\
\text { type }\end{array}$ & $\begin{array}{l}\text { Extent } \\
\text { (sq m) }\end{array}$ & Content & $\begin{array}{l}\text { Ceramic } \\
\text { series }\end{array}$ & Age BP & References \\
\hline WBR040 & Deva & coastal flat & no estimate & pot sherd & Late & 2750 & Sand et al. 2013 \\
\hline \multicolumn{8}{|c|}{ South coast } \\
\hline SNA019 & Anse Vata & coastal flat & - & pot sherd & ?Late & - & Frimigacci 1975 \\
\hline \multicolumn{8}{|c|}{ Ile des Pines } \\
\hline KV0001 & Kapume & $\begin{array}{l}\text { coastal } \\
\text { dune }\end{array}$ & - & pot sherd & ?Late & - & Golson 1962 \\
\hline KV0003 & $\begin{array}{l}\text { St Maurice- } \\
\text { Vatcha }\end{array}$ & $\begin{array}{l}\text { coastal } \\
\text { dune }\end{array}$ & 18000 & full range & $\begin{array}{l}\text { Early } \\
\text { to Late }\end{array}$ & $2950-2700$ & Sand 1999 \\
\hline KGJ004 & Gadji (1) & coastal flat & - & pot sherd & ?Late & & Frimigacci 1975 \\
\hline \multicolumn{8}{|c|}{ East coast } \\
\hline EHI050 & Dowalwoue & $\begin{array}{l}\text { coastal } \\
\text { sand }\end{array}$ & - & pot sherd & ?Late & - & $\begin{array}{l}\text { Gifford and Shutler } \\
1956\end{array}$ \\
\hline SUN014 & Witpwe & coastal flat & - & pot sherd & Late & - & Sand and Ouetcho 1992 \\
\hline STY007 & Pwekina & coastal flat & 5000 & pottery & Late & - & Sand and Ouetcho 1992 \\
\hline STY015 & Goro & coastal flat & $>10000$ & full range & $\begin{array}{l}\text { Early } \\
\text { to Late }\end{array}$ & - & Sand et al. 2000 \\
\hline No code & Kouaoua & coastal flat & no estimate & pottery & ?Late & - & Sand 2010 \\
\hline No code & Pouebo & unknown & no estimate & pottery & Early & - & Chiu et al. 2016 \\
\hline \multicolumn{8}{|c|}{ Loyalty Islands } \\
\hline LP0020 & Patho & coastal flat & $>2000$ & full range & $\begin{array}{l}\text { ?Early } \\
\text { to Late }\end{array}$ & - & $\begin{array}{l}\text { Sémah and Galipaud } \\
1992\end{array}$ \\
\hline LP0023 & Kurin & coastal flat & $>5000$ & full range & $\begin{array}{l}\text { Early } \\
\text { to Late }\end{array}$ & - & Sand et al. 2002 \\
\hline LLI002 & Hnaеo & $\begin{array}{l}\text { coastal } \\
\text { dune }\end{array}$ & - & full range & $\begin{array}{l}\text { Early } \\
\text { to Late }\end{array}$ & - & Sand et al. 1999b \\
\hline LWT008 & Hnajoisisi & rock shelter & 50 & full range & Late & $2800-2750$ & Sand 1998b \\
\hline LWT054 & Keny & $\begin{array}{l}\text { coastal } \\
\text { dune }\end{array}$ & $>20000$ & full range & $\begin{array}{l}\text { ?Early } \\
\text { to Late }\end{array}$ & $2950-2750$ & Sand 1998b \\
\hline LUV081 & Wadrilla & $\begin{array}{l}\text { coastal } \\
\text { sand }\end{array}$ & - & pottery & - & - & Sand et al. 1999b \\
\hline LTD825 & Namara & $\begin{array}{l}\text { coastal } \\
\text { beach }\end{array}$ & - & pottery & - & - & Sand et al. 2010 \\
\hline \multicolumn{8}{|c|}{ FIJI ISLANDS } \\
\hline \multicolumn{8}{|c|}{ Western Islands } \\
\hline Y2-25 & $\begin{array}{l}\text { Yalobi, Waya } \\
\text { Island } \\
\end{array}$ & coastal flat & - & full range & Late & $2800-2400$ & Hunt et al. 1999 \\
\hline K27-11 & \begin{tabular}{|l} 
Tavua, Tavua \\
Island \\
\end{tabular} & coastal flat & - & full range & Late & $2850-2750$ & Cochrane et al. 2011 \\
\hline \multicolumn{8}{|c|}{ Southern Islands } \\
\hline No code & $\begin{array}{l}\text { Unlocalised, } \\
\text { Vatulele Island }\end{array}$ & unknown & - & pottery & Late & - & Ewin 1995 \\
\hline BQ178A & $\begin{array}{l}\text { Nadawa, Beqa } \\
\text { Island }\end{array}$ & coastal flat & - & pottery & Late & - & Crosby 1988 \\
\hline BQ175A & $\begin{array}{l}\text { Kulu, Beqa } \\
\text { Island }\end{array}$ & coastal flat & $<1000$ & full range & Late & - & $\begin{array}{l}\text { Anderson and Clark } \\
1999\end{array}$ \\
\hline $93 \mathrm{~A}$ & $\begin{array}{l}\text { Melabe, Beqa } \\
\text { Island }\end{array}$ & coastal flat & - & pottery & Late & - & Crosby 1988 \\
\hline BQ132 & $\begin{array}{l}\text { Beqa, Beqa } \\
\text { Island }\end{array}$ & coastal flat & - & pottery & Late & - & Crosby 1988 \\
\hline
\end{tabular}




\begin{tabular}{|c|c|c|c|c|c|c|c|}
\hline Code & Location name & $\begin{array}{l}\text { Locale } \\
\text { type }\end{array}$ & $\begin{array}{l}\text { Extent } \\
(\mathrm{sq} \mathrm{m})\end{array}$ & Content & $\begin{array}{l}\text { Ceramic } \\
\text { series }\end{array}$ & Age BP & References \\
\hline UG1-2 & $\begin{array}{l}\text { Ugaga, Ugaga } \\
\text { Island }\end{array}$ & islet & $100-500$ & full range & Late & $2800-2300$ & Clark 2009 \\
\hline No code & $\begin{array}{l}\text { Nalotu Water } \\
\text { Tank, Kadavu } \\
\text { Island }\end{array}$ & $\begin{array}{l}\text { inland, } \\
\text { creek }\end{array}$ & - & pottery & Late & - & $\begin{array}{l}\text { Burley and Balenaivalu } \\
2012\end{array}$ \\
\hline No code & $\begin{array}{l}\text { Tiliva Resort, } \\
\text { Kadavu Island }\end{array}$ & back beach & 500 & pottery & Late & - & $\begin{array}{l}\text { Burley and Balenaivalu } \\
2012\end{array}$ \\
\hline No code & $\begin{array}{l}\text { Waisomo } \\
\text { Makawa, 0no } \\
\text { Island }\end{array}$ & coastal flat & - & pottery & Late & - & $\begin{array}{l}\text { Burley and Balenaivalu } \\
2012\end{array}$ \\
\hline \multicolumn{8}{|c|}{ Viti Levu Island } \\
\hline $\mathrm{VL} 1 / 1$ & Natunuku & $\begin{array}{l}\text { coastal } \\
\text { dune }\end{array}$ & 1000 & full range & $\begin{array}{l}\text { Middle } \\
\text { to Late }\end{array}$ & $2900-2300$ & Davidson et al. 1990 \\
\hline VL16/81 & Yanuca & rock shelter & $1000-3000$ & full range & $\begin{array}{l}\text { Middle } \\
\text { to Late }\end{array}$ & - & Hunt 1980 \\
\hline No code & Qara-I-Oso II & $\begin{array}{l}\text { inland } \\
\text { shelter }\end{array}$ & 150 & pot sherd & Late & - & Anderson et al. 2000 \\
\hline VL15/1 & Natadola Bay & & - & pot sherd & Late & - & Palmer 1966 \\
\hline VL16/22 & Naqarai & $\begin{array}{l}\text { coastal } \\
\text { dune }\end{array}$ & - & pottery & Late & - & Hunt 1980 \\
\hline VL16/1 & Sigatoka & $\begin{array}{l}\text { coastal } \\
\text { dune }\end{array}$ & - & pottery & Late & $2550-2700$ & $\begin{array}{l}\text { Petchey 1995; Burley } \\
\text { and Connaughton } 2010\end{array}$ \\
\hline No code & Bourewa & $\begin{array}{l}\text { sandspit/ } \\
\text { beach }\end{array}$ & 12500 & full range & $\begin{array}{l}\text { Middle } \\
\text { to Late }\end{array}$ & $3000-2700$ & Nunn 2007 \\
\hline No code & Rove Beach & coastal flat & - & pottery & Late & - & Kumar et al. 2004 \\
\hline No code & Waikereiгa Вау & coastal flat & - & $\begin{array}{l}\text { surface } \\
\text { sherds }\end{array}$ & Late & - & Nunn 2007 \\
\hline No code & $\begin{array}{l}\text { Jugendars Farm } \\
\text { Bay }\end{array}$ & coastal flat & - & $\begin{array}{l}\text { surface } \\
\text { sherds }\end{array}$ & Late & - & Nunn 2007 \\
\hline No code & $\begin{array}{l}\text { Tomato Patch } \\
\text { Bay }\end{array}$ & coastal flat & - & $\begin{array}{l}\text { surface } \\
\text { sherds }\end{array}$ & Late & - & Nunn 2007 \\
\hline No code & Qoqo Island & tombolo & 5000 & full range & $\begin{array}{l}\text { Middle } \\
\text { to Late }\end{array}$ & $2850-2650$ & Nunn et al. 2006 \\
\hline No code & Navutulevu & coastal flat & - & $\begin{array}{l}\text { surface } \\
\text { sherds }\end{array}$ & - & - & Kumar et al. 2004 \\
\hline No code & Qaqaruku & rock shelter & - & $\begin{array}{l}\text { surface } \\
\text { sherds }\end{array}$ & Late & - & Kumar 2002 \\
\hline \multicolumn{8}{|c|}{ Central Islands (Koro Sea) } \\
\hline No code & \begin{tabular}{|l} 
Saulevu, \\
Moturiki Island
\end{tabular} & islet & - & pottery & Late & - & Nunn 1999 \\
\hline No code & $\begin{array}{l}\text { Naitabale, } \\
\text { Moturiki Island }\end{array}$ & back beach & 300 & full range & $\begin{array}{l}\text { Middle } \\
\text { to Late }\end{array}$ & $2900-2700$ & Nunn et al. 2007 \\
\hline $\mathrm{VL} 21 / 5$ & $\begin{array}{l}\text { Naigani, Naigani } \\
\text { Island }\end{array}$ & coastal flat & $1000-3000$ & full range & Middle & $2900-2700$ & $\begin{array}{l}\text { Best 2002; Irwin et al. } \\
2011\end{array}$ \\
\hline No code & $\begin{array}{l}\text { Vagariki, Yadua } \\
\text { Island }\end{array}$ & coastal flat & - & pottery & Late & 2600 & Nunn et al. 2005 \\
\hline No code & $\begin{array}{l}\text { Taviya, 0valau } \\
\text { Island }\end{array}$ & coastal flat & - & $\begin{array}{l}\text { surface } \\
\text { sherds }\end{array}$ & - & - & Nunn et al. 2004 \\
\hline \multicolumn{8}{|c|}{ Northern Islands } \\
\hline No code & $\begin{array}{l}\text { Yacata, Yacata } \\
\text { Island }\end{array}$ & unknown & - & pottery & Late & - & Parke 2000 \\
\hline
\end{tabular}




\begin{tabular}{|c|c|c|c|c|c|c|c|}
\hline Code & Location name & $\begin{array}{l}\text { Locale } \\
\text { type }\end{array}$ & $\begin{array}{l}\text { Extent } \\
(\mathrm{sq} \mathrm{m})\end{array}$ & Content & $\begin{array}{l}\text { Ceramic } \\
\text { series }\end{array}$ & Age BP & References \\
\hline No code & $\begin{array}{l}\text { Vaturekuka, } \\
\text { Vanua Levu } \\
\text { Island }\end{array}$ & riverbank & - & $\begin{array}{l}\text { pottery and } \\
\text { lithics }\end{array}$ & Late & - & Parke 2000 \\
\hline No code & $\begin{array}{l}\text { Nukubalavu, } \\
\text { Vanua Levu } \\
\text { Island }\end{array}$ & back beach & 500 & full range & Late & 2800 & Jones pers. comm. \\
\hline No code & $\begin{array}{l}\text { Vorovoro, } \\
\text { Vorovoro Island }\end{array}$ & tombolo & 500 & full range & Middle & 3000 & Burley 2012 \\
\hline No code & $\begin{array}{l}\text { Ligaulevu, Mali } \\
\text { Island }\end{array}$ & coastal flat & - & pottery & Late & - & Burley notes \\
\hline No code & $\begin{array}{l}\text { Kavewa, Kavewa } \\
\text { Island }\end{array}$ & coastal flat & 750 & full range & Middle & - & $\begin{array}{l}\text { Burley et al. this } \\
\text { volume }\end{array}$ \\
\hline CIK006 & $\begin{array}{l}\text { Naselala, } \\
\text { Cikobia-i-Ra } \\
\text { Island }\end{array}$ & coastal flat & - & full range & Late & $2800-2400$ & Sand et al. 1999a \\
\hline \multicolumn{8}{|l|}{ Lau Group } \\
\hline No code & $\begin{array}{l}\text { Susui, } \\
\text { Bureniwaqa } \\
\end{array}$ & coastal flat & - & pottery & Late & - & $\begin{array}{l}\text { Nunn and Matararaba } \\
2000\end{array}$ \\
\hline No code & $\begin{array}{l}\text { Cikobia-i-Lau, } \\
\text { Cikobia-i-Lau } \\
\text { Island }\end{array}$ & coastal flat & - & pottery & Late & - & $\begin{array}{l}\text { Nunn and Matararaba } \\
2000\end{array}$ \\
\hline No code & $\begin{array}{l}\text { Votua, Mago } \\
\text { Island }\end{array}$ & coastal flat & $1000-3000$ & full range & Late & $2800-2600$ & Clark 2009 \\
\hline No code & $\begin{array}{l}\text { Sovanibeka, } \\
\text { Mago Island }\end{array}$ & rock shelter & 30 & pottery & Late & $2700-2300$ & Clark and Hope 1997 \\
\hline $101 / 7 / 197$ & $\begin{array}{l}\text { Lakeba, Lakeba } \\
\text { Island }\end{array}$ & rock shelter & 80 & full range & $\begin{array}{l}\text { Middle } \\
\text { to Late }\end{array}$ & $2800-2700$ & Best 1984 \\
\hline 101/7/196 & $\begin{array}{l}\text { Qaranipuqa- } \\
\text { Wakea, Lakeba } \\
\text { Island }\end{array}$ & coastal flat & 15000 & full range & $\begin{array}{l}\text { Middle } \\
\text { to Late }\end{array}$ & $2800-2700$ & Best 1984 \\
\hline No code & $\begin{array}{l}\text { Namuka, } \\
\text { Namuka Island }\end{array}$ & unknown & - & pottery & - & - & Best pers. comm. \\
\hline No code & $\begin{array}{l}\text { Komo, Komo } \\
\text { Island }\end{array}$ & unknown & - & pottery & - & - & Best pers. comm. \\
\hline No code & $\begin{array}{l}\text { Unlocalised (2), } \\
\text { Moce Island }\end{array}$ & unknown & - & pottery & - & - & Best 1984 \\
\hline No code & $\begin{array}{l}\text { Fulaga, Fulaga } \\
\text { Island }\end{array}$ & unknown & - & pottery & - & - & Best 1984 \\
\hline To31/1 & $\begin{array}{l}\text { Udu, Totoya } \\
\text { Island }\end{array}$ & coastal flat & - & pottery & Late & - & Clark and Cole 1997 \\
\hline To31/2 & $\begin{array}{l}\text { Lawaki Levu, } \\
\text { Totoya Island }\end{array}$ & coastal flat & - & pottery & Late & - & Clark and Cole 1997 \\
\hline To31/3 & $\begin{array}{l}\text { Waroke, Totoya } \\
\text { Island }\end{array}$ & coastal flat & - & pottery & Late & - & Clark and Cole 1997 \\
\hline No code & $\begin{array}{l}\text { 0no-i-Lau (3), } \\
\text { 0ni-i-Lau Island }\end{array}$ & coastal flat & - & pottery & - & - & Best 1984 \\
\hline No code & $\begin{array}{l}\text { Na Masimasi, } \\
\text { Nayau Island }\end{array}$ & coastal flat & - & full range & Late & - & 0'Day et al. 2004 \\
\hline No code & $\begin{array}{l}\text { Vulago, Nayau } \\
\text { Island }\end{array}$ & coastal flat & - & full range & Late & - & 0'Day et al. 2004 \\
\hline
\end{tabular}




\begin{tabular}{|c|c|c|c|c|c|c|c|}
\hline Code & Location name & $\begin{array}{l}\text { Locale } \\
\text { type }\end{array}$ & $\begin{array}{l}\text { Extent } \\
(\mathrm{sq} \mathrm{m})\end{array}$ & Content & $\begin{array}{l}\text { Ceramic } \\
\text { series }\end{array}$ & Age BP & References \\
\hline \multicolumn{8}{|l|}{ TONGA } \\
\hline \multicolumn{8}{|l|}{ Tongatapu } \\
\hline To.2/TO-NK-2 & Nukuleka & coastal flat & 2500 & full range & $\begin{array}{l}\text { Middle/ } \\
\text { Late }\end{array}$ & $2850-2650$ & $\begin{array}{l}\text { Poulsen 1987; Burley } \\
\text { et al. 2010, } 2012\end{array}$ \\
\hline No code & Hopoate & coastal flat & 2000 & full range & $\begin{array}{l}\text { Middle/ } \\
\text { Late }\end{array}$ & $2850-2650$ & Burley 2016 \\
\hline No code & Talasiu & $\begin{array}{l}\text { back beach } \\
\text { ridge }\end{array}$ & 450 & full range & Late & $2700-2650$ & Clark et al. 2015 \\
\hline No code & Tatakamotonga & $\begin{array}{l}\text { back beach } \\
\text { ridge }\end{array}$ & - & pottery & Late & - & Burley et al. 2001 \\
\hline No code & $\begin{array}{l}\text { Captain Cook } \\
\text { Landing } \\
\end{array}$ & $\begin{array}{l}\text { back beach } \\
\text { ridge }\end{array}$ & - & pottery & Late & - & Burley notes \\
\hline No code & Tinopai & coastal flat & - & pottery & Late & $2750-2650$ & Burley notes \\
\hline No code & Kauvai 1 & coastal flat & - & pottery & $\begin{array}{l}\text { ?Middle/ } \\
\text { Late }\end{array}$ & - & Burley notes \\
\hline No code & Kauvai 2 & $\begin{array}{l}\text { back beach } \\
\text { ridge }\end{array}$ & 500 & full range & Late & $2750-2650$ & Burley notes \\
\hline No code & Nukuhetulu & $\begin{array}{l}\text { back beach } \\
\text { ridge }\end{array}$ & - & pottery & Late & - & Burley et al. 2001 \\
\hline No code & Kanatea Island & $\begin{array}{l}\text { back beach } \\
\text { ridge }\end{array}$ & - & pottery & Late & - & Burley notes \\
\hline T0.5/T0-Pe-5 & Ha'ateiho & $\begin{array}{l}\text { back beach } \\
\text { ridge }\end{array}$ & - & full range & Late & $2800-2650$ & $\begin{array}{l}\text { Poulsen 1987; Burley } \\
\text { et al. } 2001\end{array}$ \\
\hline No code & \begin{tabular}{|l} 
Uluaki \\
(Golf Course)
\end{tabular} & $\begin{array}{l}\text { back beach } \\
\text { ridge }\end{array}$ & 750 & full range & Late & $2750-2650$ & Burley notes \\
\hline $\begin{array}{l}\text { T0.3 \& 4/ } \\
\text { T0-Pe-3 }\end{array}$ & $\begin{array}{l}\text { Pea, Taufa'ahau } \\
\text { Road } \\
\end{array}$ & coastal flat & 2150 & full range & Late & $2750-2650$ & Poulsen 1987 \\
\hline To.1 & Pea School Yard & coastal flat & 4300 & full range & Late & $2800-2650$ & Poulsen 1987 \\
\hline T0.6/T0-Pe-6 & Tufu Mahina & $\begin{array}{l}\text { back beach } \\
\text { ridge }\end{array}$ & 1500 & full range & Late & $2600-2300$ & Poulsen 1987 \\
\hline T0-Pe-28 & Vaiola Hospital & $\begin{array}{l}\text { back beach } \\
\text { ridge }\end{array}$ & - & pottery & Late & - & $\begin{array}{l}\text { Spennemann 1989; } \\
\text { Burley et al. } 2001\end{array}$ \\
\hline No code & Hofoa & $\begin{array}{l}\text { back beach } \\
\text { ridge }\end{array}$ & - & pottery & Late & - & Burley notes \\
\hline TO-Nu-2 & Fire Station & coastal flat & 2500 & full range & Late & $2750-2650$ & $\begin{array}{l}\text { Spennemann 1989; } \\
\text { Burley et al. } 2001\end{array}$ \\
\hline TO-Nu-12 & Unga Road & coastal flat & - & pottery & Late & - & $\begin{array}{l}\text { Spennemann 1989; } \\
\text { Burley et al. } 2001\end{array}$ \\
\hline TO-Nu-8 & Mangaia Mound & coastal flat & 500 & full range & Late & $2750-2650$ & Poulsen 1987 \\
\hline No code & Puke & $\begin{array}{l}\text { back beach } \\
\text { ridge }\end{array}$ & 500 & full range & Late & $2700-2600$ & Burley notes \\
\hline No code & Sia'atoutai Flats & coastal flat & - & pottery & Late & - & Burley notes \\
\hline \multicolumn{8}{|c|}{ Ha'apai Group } \\
\hline No code & \begin{tabular}{|l} 
Fakatafenga, \\
Tungua
\end{tabular} & coastal flat & 500 & pottery & Late & - & Burley et al. 1999 \\
\hline No code & $\begin{array}{l}\text { Ha'afeva, } \\
\text { Mele Havea }\end{array}$ & coastal flat & 750 & pottery & Late & - & Burley et al. 1999 \\
\hline No code & Vaipuna, 'Uiha & coastal flat & 750 & pottery & Late & $2800-2500$ & Burley et al. 1999 \\
\hline No code & $\begin{array}{l}\text { Tongoleleka, } \\
\text { Lifuka }\end{array}$ & $\begin{array}{l}\text { back beach } \\
\text { ridge }\end{array}$ & 1500 & full range & Late & $2800-2600$ & Burley et al. 1999 \\
\hline
\end{tabular}




\begin{tabular}{|c|c|c|c|c|c|c|c|}
\hline Code & Location name & \begin{tabular}{|l} 
Locale \\
type
\end{tabular} & $\begin{array}{l}\text { Extent } \\
(\mathrm{sq} \mathrm{m}) \\
\end{array}$ & Content & $\begin{array}{l}\text { Ceramic } \\
\text { series }\end{array}$ & Age BP & References \\
\hline No code & Faleloa, Foa & $\begin{array}{l}\text { back beach } \\
\text { ridge }\end{array}$ & 750 & full range & Late & $2650-2550$ & Burley et al. 1999 \\
\hline No code & Pukotala, Ha'ano & $\begin{array}{l}\text { back beach } \\
\text { ridge }\end{array}$ & 100 & pottery & Late & $2700-2600$ & Burley et al. 1999 \\
\hline \multicolumn{8}{|c|}{ Vava'u Group } \\
\hline No code & $\begin{array}{l}\text { Vuna, } \\
\text { Pangaimotu } \\
\text { Island }\end{array}$ & $\begin{array}{l}\text { coastal } \\
\text { dune }\end{array}$ & 1500 & full range & Late & $2750-2600$ & $\begin{array}{l}\text { Burley 2007; Burley } \\
\text { and Connaughton } 2007\end{array}$ \\
\hline No code & Ofu, Ofu Island & coastal flat & 1500 & full range & Late & $2750-2600$ & $\begin{array}{l}\text { Burley 2007; Burley } \\
\text { and Connaughton } 2007\end{array}$ \\
\hline No code & $\begin{array}{l}\text { '0tea, Kapa } \\
\text { Island }\end{array}$ & $\begin{array}{l}\text { back beach } \\
\text { ridge }\end{array}$ & 800 & full range & Late & $2750-2600$ & \begin{tabular}{|l|} 
Burley 2007; Burley \\
and Connaughton 2007
\end{tabular} \\
\hline No code & $\begin{array}{l}\text { Falevai, Kapa } \\
\text { Island } \\
\end{array}$ & $\begin{array}{l}\text { back beach } \\
\text { ridge }\end{array}$ & 500 & full range & Late & $2700-2600$ & \begin{tabular}{|l|} 
Burley 2007; Burley \\
and Connaughton 2007
\end{tabular} \\
\hline No code & $\begin{array}{l}\text { Mafana, Mafana } \\
\text { Island }\end{array}$ & coastal flat & 400 & pottery & Late & - & Burley 2007 \\
\hline \multicolumn{8}{|c|}{ Niuatoputapu } \\
\hline NT-90 & Lolokoka & coastal flat & 3000 & pottery & Late & $2750-2600$ & Kirch 1988 \\
\hline \multicolumn{8}{|l|}{ SAMOA } \\
\hline \multicolumn{8}{|l|}{ Upolu } \\
\hline No code & Mulifanua & $\begin{array}{l}\text { submerged } \\
\text { beach }\end{array}$ & 6000 & pottery & ?Early & 2750 & $\begin{array}{l}\text { Dickinson and Green } \\
1998\end{array}$ \\
\hline \multicolumn{8}{|l|}{ WALLIS } \\
\hline MU021 & Utuleve & $\begin{array}{l}\text { coastal } \\
\text { sand }\end{array}$ & 10000 & full range & \begin{tabular}{|l} 
Early \\
to Late
\end{tabular} & $2800-2750$ & Sand 1998c \\
\hline MU046 & Utuleve & coastal flat & 1000 & full range & \begin{tabular}{|l} 
Early \\
to Late \\
\end{tabular} & - & Frimigacci 2000 \\
\hline $\mathrm{HI} 012$ & Utupoa & coastal flat & - & pottery & Late & - & Frimigacci 2000 \\
\hline \multicolumn{8}{|l|}{ FUTUNA } \\
\hline SI001 & Asipani & coastal flat & $>3000$ & full range & Late & - & Sand 1993 \\
\hline
\end{tabular}

Source: See references throughout table.

\section{References}

Anderson, A. and G. Clark 1999. The age of the Lapita settlement in Fiji. Archaeology in Oceania 34:31-39. doi.org/10.1002/j.1834-4453.1999.tb00424.x.

Anderson, A., G. Clark and T. Worthy 2000. An inland Lapita site in Fiji. Journal of the Polynesian Society 109:311-316.

Anderson, A., S. Bedford, G. Clark, I. Lilley, C. Sand, G. Summerhayes and R. Torrence 2001. An Inventory of Lapita sites containing dentate-stamped pottery. In G. Clark, A. Anderson and T. Sorovi-Vunidilo (eds), The archaeology of Lapita dispersal in Oceania: Papers from the Fourth Lapita Conference, June 2000, Canberra, Australia, pp. 1-14. Terra Australis 17. Pandanus Books, The Australian National University, Canberra.

Anggraeni, T. Simanjuntak, P. Bellwood and P. Piper 2014. Neolithic foundations in the Karama Valley, West Sulawesi, Indonesia. Antiquity 88(341):740-756. doi.org/10.1017/s0003598x00050663. 
Anson, D., R. Walter and R.C. Green 2005. A revised and redated event phase sequence for the Reber-Rakival Lapita site, Watom Island, East New Britain Province, Papua New Guinea. University of Otago Studies in Prehistoric Anthropology 20. University of Otago, Dunedin.

Aoyagi, Y., M.L Aguilera, H. Ogawa and K. Tanaka 1993. Excavations of Hill Top Site, Magapit Shell Midden, in Lal-Lo Shell Middens, Northern Luzon, Philippines. Man and Culture in Oceania 9:127-155.

Azis, N., C. Reepmeyer, G. Clark, Sriwigati and D.A. Tanudirjo 2018. Mansiri in North Sulawesi: A new dentate-stamped pottery site in Island Southeast Asia. In S. O'Connor, D. Bulbeck and J. Meyer (eds), The archaeology of Sulawesi: Current research on the Pleistocene to the Historic period, pp. 191-205. Terra Australis 48. ANU Press, Canberra. doi.org/10.22459/TA48.11.2018.

Baret, D., J. Bole, A. Ouetcho and C. Sand 2000. Etude de potentiel et pré-inventaire des ressources patrimoniales du milieu. Unpublished. Projet Koniambo: Etude Environnemental de Base, Nouméa.

Barp, F., D. Baret, S. Domergue and M.-K. Haluathr 2006. Projet Koniambo. Etude Archéologique Phase 3. Rapport Final d'Opération. Unpublished. Rapport interne Falconbridge, Nouméa.

Bedford, S. 2003. The timing and nature of Lapita colonisation in Vanuatu: The haze begins to clear. In C. Sand (ed.), Pacific archaeology: Assessments and prospects. Proceedings of the conference for the 50th anniversary of the first Lapita excavation, Kone-Nouméa, 2002, pp. 147-158. Les cahiers de l'archéologie en Nouvelle-Calédonie 15. Département Archéologie, Service des Musées et du Patrimoine de Nouvelle-Calédonie, Nouméa.

Bedford, S. 2006a. Pieces of the Vanuatu puzzle: Archaeology of the north, south and centre. Terra Australis 23. Pandanus Books, The Australian National University, Canberra. doi.org/10.22459/ PVP.02.2007.

Bedford, S. 2006b. The Pacific's earliest painted pottery: An added layer of intrigue to the Lapita debate and beyond. Antiquity 80:544-557. doi.org/10.1017/S0003598X00094023.

Bedford, S. and J.-C. Galipaud 2010. Chain of islands: Lapita in the north of Vanuatu. In C. Sand and S. Bedford (eds), Lapita: Ancêtres Océaniens/Oceanic ancestors, pp. 122-137. Museé du quai Branly and Somogy, Paris.

Bedford, S. and C. Sand 2007. Lapita and Western Pacific settlement: Progress, prospects and persistent problems. In S. Bedford, C. Sand and S.P. Connaughton (eds), Oceanic explorations: Lapita and Western Pacific settlement, pp. 1-16. Terra Australis 26. ANU E Press, Canberra. doi.org/10.22459/ TA26.2007.

Bedford, S. and M. Spriggs 2008. Northern Vanuatu as a Pacific crossroads: The archaeology of discovery, interaction, and the emergence of the 'ethnographic present'. Asian Perspectives 47(1):95-120. doi.org/ 10.1353/asi.2008.0003.

Bedford, S. and M. Spriggs 2014. The archaeology of Vanuatu: 3000 years of history across islands of ash and coral. In E. Cochrane and T. Hunt (eds), The Oxford handbook of prehistoric Oceania. Oxford University Press, Oxford. doi.org/10.1093/oxfordhb/9780199925070.013.015.

Bedford, S., M. Spriggs, M. Wilson and R. Regenvanu 1998. The Australian National University-National Museum of Vanuatu Archaeology Project, 1994-7: A preliminary report on the establishment of cultural sequences and rock art research. Asian Perspectives 37(2):165-193. doi.org/10.1002/j.18344461.1999.tb02986.x.

Bedford, S., M. Spriggs, H. Buckley, F. Valentin, R. Regenvanu and M. Abong 2010. A cemetery of first settlement: The site of Teouma, South Efate, Vanuatu. In C. Sand and S. Bedford (eds), Lapita: Ancêtres Océaniens/Oceanic ancestors, pp. 140-161. Museé du quai Branly and Somogy, Paris. 
Bedford, S., H. Buckley, F. Valentin, N. Tayles and N. Longga 2011. Lapita burials, a new Lapita cemetery and Post-Lapita burials from Malakula, northern Vanuatu, Southwest Pacific. Journal of Pacific Archaeology 2(2):26-48.

Bedford, S., M. Spriggs and R. Shing 2016. 'By all means let us complete the exercise': The 50-year search for Lapita on Aneityum, southern Vanuatu and implications for other 'gaps' in the Lapita distribution. Archaeology in Oceania 51:122-130. doi.org/10.1002/arco.5100.

Bellwood, P. and P. Koon 1989. 'Lapita colonists leave boats unburned!' The question of Lapita links with Island Southeast Asia. Antiquity 63(240):613-622. doi.org/10.1017/S0003598X00076572.

Best, S. 1984. Lakeba: The prehistory of a Fijian island. Unpublished PhD thesis, University of Auckland, Auckland.

Best, S. 2002. Lapita: A view from the east. New Zealand Archaeological Association Monograph 24. New Zealand Archaeological Association, Auckland.

Burley, D.V. 2007. In search of Lapita and Polynesian plainware settlements in Vava'u, Kingdom of Tonga. In S. Bedford, C. Sand and S.P. Connaughton (eds), Oceanic explorations: Lapita and Western Pacific settlement, pp. 187-198. Terra Australis 26. ANU E Press, Canberra. doi.org/10.22459/ TA26.2007.

Burley, D.V. 2012. Exploration as a strategic process in the Lapita settlement of Fiji: The implications of Vorovoro Island. Journal of Pacific Archaeology 3(1):22-34.

Burley, D.V. 2016. Reconsideration of sea level and landscape for first Lapita settlement at Nukuleka, Kingdom of Tonga. Archaeology in Oceania 51:84-90. doi.org/10.1002/arco.5087.

Burley, D.V. and J. Balenaivalu 2012. Kadavu archaeology: First insights from a preliminary survey. Domodomo 25(1 and 2):13-36.

Burley, D.V. and S.P. Connaughton 2007. First Lapita settlement and its chronology in Vava'u, Kingdom of Tonga. Radiocarbon 49(1):131-137. doi.org/10.1017/S0033822200041965.

Burley, D.V. and S.P. Connaughton 2010. Completing the story: A Late Lapita dentate stamped pot from Sigatoka, Fiji. Archaeology in Oceania 45:130-132. doi.org/10.1002/j.1834-4453.2010. tb00090.x.

Burley, D.V., D.E. Nelson and R. Shutler Jr 1999. A radiocarbon chronology for the Eastern Lapita frontier in Tonga. Archaeology in Oceania 34(2):59-70. doi.org/10.1002/j.1834-4453.1999. tb00429.x.

Burley, D.V., W.R. Dickinson, A. Barton and R. Shutler 2001. Lapita on the periphery: New data on old problems in the Kingdom of Tonga. Archaeology in Oceania 36(2):89-104. doi.org/10.1002/j.18344453.2001.tb00481.x.

Burley, D.V., A. Barton, W.R. Dickinson, S.P. Connaughton and K. Taché 2010. Nukuleka as a founder colony for west Polynesian settlement: New insights from recent excavations. Journal of Pacific Archaeology 1(2):128-144.

Burley, D.V., M.I. Weisler and J.-x. Zhao 2012. High precision U/Th dating of first Polynesian settlement. PLoS ONE 7(11):e48769. doi.org/10.1371/journal.pone.0048769.

Carson, M.T. 2014. First settlement of Remote Oceania: Earliest sites in the Mariana Islands. Springer, Heidelberg. doi.org/10.1007/978-3-319-01047-2.

Carson, M.T. 2018. Archaeology of Pacific Oceania: Inhabiting a sea of islands. Taylor and Francis, Abingdon. 
Carson, M.T., H.-C. Hung, G.R. Summerhayes and P. Bellwood 2013. The pottery trail from Southeast Asia to Remote Oceania. The Journal of Island and Coastal Archaeology 8(1):17-36. doi.org/10.1080/ 15564894.2012.726941.

Cath-Garling, S. 2017. Evolutions or revolutions? Interaction and transformation at the 'transition' in Island Melanesia. University of Otago Studies in Archaeology 27. University of Otago, Dunedin.

Chia, S. 2003. The prehistory of Bukit Tengkorak as a major pottery making site in Island Southeast Asia. Sabah Museum Monograph 8. Sabah Museum, Kota Kinabalu.

Chiu, S., D. Killick, C. Sand and W.R. Dickinson 2016. Connection and competition: Some early insights gained from petrographic studies of New Caledonian Lapita pottery. Archaeology in Oceania 51(2):141-149. doi.org/10.1002/arco.5093.

Clark, G. 2009. Ceramic assemblages from excavations on Viti Levu, Beqa-Ugaga and Mago Island. In G. Clark and A. Anderson (eds), The early prehistory of Fiji, pp. 259-306. Terra Australis 31. ANU E Press, Canberra. doi.org/10.22459/TA31.12.2009.11.

Clark, G. and G. Hope 1997. Preliminary report on archaeological and palaeoenvironmental investigations in Northern Lau (Mago, Yacata-Kaibu and Vatuvara). Unpublished report to the Fiji Museum, Suva.

Clark, G., E. Grono, E. Ussher and C. Reepmeyer 2015. Early settlement and subsistence on Tongatapu, Kingdom of Tonga: Insights from a 2700-2650 cal. BP midden deposit. Journal of Archaeological Science: Reports 3:531-524. doi.org/10.1016/j.jasrep.2015.08.005.

Clark, J.T. and A.O. Cole 1997. Environmental change and human prehistory in the Central Pacific: Archaeological and palynological investigations on Totoya Island, Fiji. Unpublished report to the Fiji Museum, Suva.

Cochrane, E., I.C. Rivera-Collazo and E. Walsh 2011. New evidence for variation in colonization, cultural transmission and subsistence from Lapita (2900 BP) to the historic period in southwestern Fiji. Journal of Pacific Archaeology 2(1):40-55.

Crosby, A. 1988. Beqa: Archaeology, structure and history in Fiji. Unpublished MA thesis, University of Auckland, Auckland.

David, B., I.J. McNiven, T. Richards, S.P. Connaughton, M. Leavesley, B. Barker and C. Rowe 2011. Lapita sites in the Central Province of mainland Papua New Guinea. World Archaeology 43(4):576-593. doi.org/10.1080/00438243.2011.624720.

Davidson, J., E. Hinds, S. Holdaway and B.F. Leach 1990. The Lapita site of Natunuku, Fiji. New Zealand Journal of Archaeology 12:121-155.

Dickinson, W.R. and R.C. Green 1998 Geoarchaeological context of Holocene subsidence at the Ferry Berth Lapita site, Mulifanua, Upolu, Samoa. Geoarchaeology 13(3):239-263. doi.org/10.1002/ (SICI)1520-6548(199802)13:3<239::AID-GEA1>3.0.CO;2-5.

Doherty, M. 2007. Post-Lapita developments in the Reef-Santa Cruz Islands, Southeast Solomon Islands. Unpublished PhD thesis, University of Auckland, Auckland.

Ewin, R. 1995. Proto-Polynesian art? The cliff paintings of Vatulele. Journal of the Polynesian Society 104:23-73.

Felgate, M. 2001. A Roviana ceramic sequence and the prehistory of Near Oceania: Work in progress. In G.R. Clark, A.J. Anderson and T. Sorovi-Vunidilo (eds), The archaeology of Lapita dispersal in Oceania. Papers from the Fourth Lapita Conference, June 2000, Canberra, Australia, pp. 39-60. Terra Australis 17. Pandanus Books, The Australian National University, Canberra. 
Felgate, M. 2003. Reading Lapita in Near Oceania: Intertidal and shallow-water pottery scatters, Roviana Lagoon, New Georgia, Solomon Islands. Unpublished PhD thesis, University of Auckland, Auckland.

Frimigacci, D. 1975. La préhistoire Néo-Calédonienne. Unpublished thèse de troisième cycle, Université Paris 1, Paris.

Frimigacci, D. 2000. La préhistoire d'Uvea (Wallis). Chronologie et périodisation. Journal de la Société des Océanistes 111:135-163. doi.org/10.3406/jso.2000.2131.

Frimigacci, D. and J.-P. Siorat 1988. L'Ilot Vert site archéologique des périodes Koné et Naïa de Nouvelle-Calédonie. Journal de la Société des Océanistes 86:3-20. doi.org/10.3406/jso.1988.2839.

Galipaud, J.-C. 1988. La poterie préhistorique Néo-Calédonienne et ses implications dans l'etude du processus de peuplement du Pacifique Occidental. Unpublished PhD thesis, Université Paris 1, Paris.

Galipaud, J.-C. 1998. The Lapita site of Atanoasao Malo, Vanuatu. Field Report No. 8. ORSTOM, Port Vila.

Galipaud, J.-C. 2001. Survey of prehistoric sites in Aore. Preliminary assessment. Unpublished report to Vanuatu Cultural Centre, Port Vila.

Galipaud, J.-C. 2010. Makué and Shokraon: Earliest arrivals and cultural transformations in northern Vanuatu. In C. Sand and S. Bedford (eds), Lapita: Ancêtres Océaniens/Oceanic ancestors, pp. 138-139. Museé du quai Branly and Somogy, Paris.

Galipaud, J.-C. and M.C. Swete Kelly 2007. Makué (Aore Island, Santo, Vanuatu): A new Lapita site in the ambit of New Britain obsidian distribution. In S. Bedford, C. Sand and S.P. Connaughton (eds), Oceanic explorations: Lapita and Western Pacific settlement, pp. 151-162. Terra Australis 26. ANU E Press, Canberra. doi.org/10.22459/TA26.2007.

Galipaud, J.-C. and B. Vienne 2005. Chronologie du peuplement et réseaux d'echanges dans le Nord du Vanuatu. Mission Santo 2005. Unpublished. Rapport Préliminaire. IRD, Nouméa.

Galipaud, J.-C., C. Reepmeyer, R. Torrence, S. Kelloway and P. White 2014. Long-distance connections in Vanuatu: New obsidian characterisations for the Makué site, Aore Island. Archaeology in Oceania 49:110-116. doi.org/10.1002/arco.5030.

Garanger, J. 1972. Archéologie des Nouvelles-Hébrides: Contribution á la connaissance des Iles du Centre. Publications de la Société des Océanistes, No. 30. ORSTOM, Paris. doi.org/10.4000/books.sdo.859.

Garling, S. 2003. Tanga takes to the stage: Another model 'transitional' site? New evidence and a contribution to the 'Incised and Applied Relief Tradition' in New Ireland. In C. Sand (ed.), Pacific archaeology: Assessments and prospects. Proceedings of the conference for the 50th anniversary of the first Lapita excavation, Kone-Nouméa, 2002, pp. 213-233. Les cahiers de l'archéologie en NouvelleCalédonie 15. Département Archéologie, Service des Musées et du Patrimoine de NouvelleCalédonie, Nouméa.

Gifford, E.W. and D. Shutler Jr 1956. Archaeological excavations in New Caledonia. Anthropological Records 18(1). University of California Press, Berkeley and Los Angeles.

Golson, J. 1959. L’archéologie du Pacifique Sud: Résultats et perspectives. Journal de la Société des Océanistes 15:5-54.

Golson, J. 1962. Rapport sur les fouilles effectuées à l'Ile des Pins (Nouvelle-Calédonie), de décembre 1959 à février 1960. Etudes Melanésiennes 14-17:11-23.

Golson, J. 1992. The ceramic sequence from Lasigi. In J.-C. Galipaud (ed.), Poterie Lapita et peuplement: Actes du Colloque Lapita, pp. 155-168. ORSTOM, Nouméa. 
Gosden, C., J. Webb, B. Marshall and G.R. Summerhayes 1994. Lolmo Cave: A mid to late Holocene site, the Arawe Islands, West New Britain Province, Papua New Guinea. Asian Perspectives 33(1):97-119.

Green, R.C. 1976. Lapita sites in the Santa Cruz group. In R.C. Green and M.M. Cresswell (eds), Southeast Solomon Islands cultural history: A preliminary survey, pp. 245-265. Royal Society of New Zealand Bulletin 11. Royal Society of New Zealand, Wellington.

Green, R.C. 1979. Lapita. In J.D. Jennings (ed.), The prehistory of Polynesia, pp. 27-60. Harvard University Press, Cambridge, Mass. doi.org/10.4159/harvard.9780674181267.c3.

Green, R.C. and M. Jones 2007. The absolute age of SE-RF-6 (Ngamanie) and its relation to SE-RF-2 (Nenumbo): Two decorated Lapita sites in the southeast Solomon Islands. New Zealand Journal of Archaeology 29:5-18.

Green, R.C., M. Jones and P.J. Sheppard 2008. The reconstructed environment and absolute dating of SE-SZ-8 Lapita site on Nendö, Santa Cruz, Solomon Islands. Archaeology in Oceania 43(2):49-61. doi.org/10.1002/j.1834-4453.2008.tb00030.x.

Hedrick, J.D. 1971. Lapita style pottery from Malo Island. Journal of the Polynesian Society 80(1):5-19.

Hedrick, J.D. n.d. Archaeological investigation of Malo prehistory: Lapita settlement strategy in the northern New Hebrides. Unpublished draft PhD thesis, University of Pennsylvania, Philadelphia, PA.

Horrocks, M. and S. Bedford 2005. Microfossil analysis of Lapita deposits in Vanuatu reveals introduced Araceae (aroids). Archaeology in Oceania 40:67-74. doi.org/10.1002/j.1834-4453.2005.tb00587.x.

Hung, H.-C. 2008. Migration and cultural interaction in Southern Coastal China, Taiwan and the Northern Philippines, 3000 BC to AD 100: The early history of the Austronesian-speaking populations. Unpublished PhD thesis, The Australian National University, Canberra.

Hung, H.-C., M.T. Carson, P. Bellwood, F.Z. Campos, P.J. Piper, E. Dizon, M.J.L.A. Bolunia, M. Oxenham and Z. Chi 2011. The first settlement of Remote Oceania: The Philippines to the Marianas. Antiquity 85:909-926. doi.org/10.1017/S0003598X00068393.

Hunt, T.L. 1980. Toward Fiji's past: Archaeological research on Southwestern Viti Levu. Unpublished MA thesis, University of Auckland, Auckland.

Hunt, T.L., K.F. Aronson, E. Cochrane, J. Field, L. Humphrey and T.M. Rieth 1999. A preliminary report on archaeological research in the Yasawa Islands, Fiji. Domodomo 12:5-43.

Irwin, G., T.H. Worthy, S. Best, S. Hawkins, J. Carpenter and S. Matararaba 2011. Further investigations at the Naigani Lapita site (VL 21/5), Fiji: Excavation, radiocarbon dating and palaeofaunal extinction. Journal of Pacific Archaeology 2(2):66-78.

Kennedy, J. 1981. Lapita colonisation of the Admiralty Islands. Science 213:757-759. doi.org/10.1126/ science.213.4509.757.

Kinaston, R., S. Bedford, M. Richards, S. Hawkins, A. Gray, K. Jaouen, F. Valentin and H. Buckley 2014. Diet and human mobility from the Lapita to the Early Historic Period on Uripiv Island, Northeast Malakula, Vanuatu. PLoS ONE 9(8):e104071. doi.org/10.1371/journal.pone.0104071.

Kirch, P.V. 1988. Niuatoputapu: The prehistory of a Polynesian chiefdom. Thomas Burke Memorial Washington State Museum Monograph 5. Burke Museum, Seattle.

Kirch, P.V. 1997. The Lapita peoples: Ancestors of the Oceanic world. Blackwell, Oxford.

Kirch, P.V. (ed.) 2001. Lapita and its transformations in Near Oceania: Archaeological investigations in the Mussau Islands, Papua New Guinea, 1985-88. Volume I: Introduction, stratigraphy, chronology. Archaeological Research Facility Contribution No 59. University of California, Berkeley. 
Kirch, P.V. and T. Hunt 1988. The spatial and temporal boundaries of Lapita. In P.V. Kirch and T. Hunt (eds), Archaeology of the Lapita Cultural Complex: A critical review, pp. 9-32. Thomas Burke Memorial Museum Research Report No. 5. Burke Museum, Seattle.

Kirch, P.V. and J. Swift 2017. New AMS radiocarbon dates and a re-evaluation of the cultural sequence of Tikopia Island, southeast Solomon Islands. Journal of the Polynesian Society 126(3):313-336. doi.org/10.15286/jps.126.3.313-336.

Kirch, P.V. and D.E. Yen 1982. Tikopia: The prehistory and ecology of a Polynesian Outlier. Bernice P. Bishop Museum Bulletin 238. Bishop Museum Press, Honolulu.

Kirch, P.V., T.L. Hunt, M.I. Weisler, V.L. Butler and M.S. Allen 1991. Mussau Islands prehistory: Results of the 1985-86 excavations. In J. Allen and C. Gosden (eds), Report of the Lapita Homeland Project, pp. 144-163. Occasional Papers in Prehistory 20. Department of Prehistory, RSPacS, The Australian National University, Canberra.

Kumar, R. 2002. Discovery of a Lapita sherd inland of the northeast coast of Viti Levu Island, Fiji: Insights and implications. The University of the South Pacific, Institute of Applied Sciences Technical Report 2002/6. USP, Suva.

Kumar, R., P.D. Nunn and W.R. Dickinson 2004. The emerging pattern of earliest human settlement in Fiji: Four new Lapita sites on Viti Levu Island. Archaeology in New Zealand 47:108-117.

Lape, P. 2000. Political dynamics and religious change in the late pre-colonial Banda Islands, Eastern Indonesia. World Archaeology 32(1):138-155. doi.org/10.1080/004382400409934.

Leach, B.F. and J.M. Davidson 2008. The archaeology of Taumako: A Polynesian Outlier in the Eastern Solomon Islands. New Zealand Journal of Archaeology Special Publication, Dunedin.

Leavesley, M. and A. Sarar 2013. Diving for pottery: Lapita in Jacquinot Bay, East New Britain, Papua New Guinea. In G.R. Summerhayes and H. Buckley (eds), Pacific archaeology: Documenting the past 50,000 years, pp. 171-174. University of Otago Studies in Anthropology 25. University of Otago, Dunedin.

Lilley, I. 1991. Lapita and Post-Lapita developments in the Vitiaz Straits-West New Britain Area. Bulletin of the Indo-Pacific Prehistory Association 11:313-322. doi.org/10.7152/bippa.v11i0.11395.

Lilley, I. 2002. Lapita and Type Y pottery in the KLK site, Siassi, Papua New Guinea. In S. Bedford, C. Sand and D. Burley (eds), Fifty years in the field: Essays in honour and celebration of Richard Shutler Jr's archaeological career, pp. 79-90. New Zealand Archaeological Association Monograph 25. New Zealand Archaeological Association, Auckland.

McCoy, P.C. and P.L. Cleghorn 1988. Archaeological excavations on Santa-Cruz (Nendö), Southeast Solomon Islands: Summary report. Archaeology in Oceania 23:104-115. doi.org/10.1002/j.18344453.1988.tb00197.x.

McEldowney, H. and C. Ballard 1991. The Mouk Island site: Manus as paradox or parable in reconstructions of the Lapita cultural complex? In J. Allen and C. Gosden (eds), Report of the Lapita Homeland Project, pp. 92-102. Occasional Papers in Prehistory 20. Department of Prehistory, RSPacS, The Australian National University, Canberra.

McKern, W.C. 1929. Archaeology of Tonga. Bernice P. Bishop Museum Bulletin 60. Bishop Museum Press, Honolulu.

McNiven, I.J., W.R. Dickinson, B. David, M. Weisler, F. Von Gnielinski, M. Carter and U. Zoppi 2006. Mask Cave: Red-slipped pottery and the Australian-Papuan settlement of Zenadh Kes (Torres Strait). Archaeology in Oceania 41:49-81. doi.org/10.1002/j.1834-4453.2006.tb00610.x. 
McNiven, I.J., B. David, T. Richards, K. Aplin, B. Asmussen, J. Mialanes, M. Leavesley, P. Faulkner and S. Ulm 2011. New direction in human colonisation of the Pacific: Lapita settlement of south coast New Guinea. Australian Archaeology 72:1-6. doi.org/10.1080/03122417.2011.11690525.

McNiven, I.J., B. David, T. Richards, C. Rowe, M. Leavesley, J. Mialanes, S.P. Connaughton, B. Barker, K. Aplin, B. Asmussen, P. Faulkner and S. Ulm 2012a. Lapita on the south coast of Papua New Guinea: Challenging new horizons in Pacific archaeology. Australian Archaeology 75:16-22. doi.org/ 10.1080/03122417.2012.11681946.

McNiven, I.J., B. David, K. Aplin, J. Mialanes, B. Asmussen, S. Ulm, P. Faulkner, C. Rowe and T. Richards 2012b. Terrestrial engagements by terminal Lapita maritime specialists on the southern Papuan coast. In S.G. Haberle and B. David (eds), Peopled landscapes: Archaeological and biogeographic approaches to landscapes, pp. 121-156. Terra Australis 34. ANU E Press, Canberra. doi.org/10.22459/ TA34.01.2012.05.

Meyer, O. 1909. Funde prähistorischer Töpferei und Steinmesser auf Vuatom, Bismarck-Archipel. Anthropos 4:1093-1095.

Mialanes, J., B. David, A. Ford, T. Richards, I.J. McNiven, G.R. Summerhayes and M. Leavesley 2016. Imported obsidian at Caution Bay, south coast of Papua New Guinea: Cessation of long-distance procurement c. 1,900 cal. BP. Australian Archaeology 82(3):248-262. doi.org/10.1080/03122417. 2016.1252079.

Noury, A. and J.-C. Galipaud 2011. Les Lapita: Nomades du Pacifique. IRD Éditions, Marseille. doi.org/10.4000/books.irdeditions.653.

Nunn, P.V. 1999. Lapita pottery from Moturiki Island, Central Fiji. Archaeology in New Zealand 42:309-313.

Nunn, P.V. 2007. Echoes from a distance: Research into the Lapita occupation of the Rove Peninsula, Southwest Viti Levu, Fiji. In S. Bedford, C. Sand and S.P. Connaughton (eds), Oceanic explorations: Lapita and Western Pacific settlement, pp. 163-176. Terra Australis 26. ANU E Press, Canberra. doi.org/10.22459/TA26.2007.

Nunn, P. and T.A. Heorake 2009. Understanding the place properly: Palaeogeography of selected Lapita sites in the western tropical Pacific islands and its implications. In P. Sheppard, G.R. Summerhayes and T. Thomas (eds), Lapita: Ancestors and descendants, pp. 235-254. New Zealand Archaeological Association Monograph 28. New Zealand Archaeological Association, Auckland.

Nunn, P.D. and S. Matararaba 2000. New finds of Lapita pottery in northeast Fiji. Archaeology in Oceania 35:92-93. doi.org/10.1002/j.1834-4453.2000.tb00459.x.

Nunn, P.D., R. Kumar, S. Matararaba, T. Ishimura, J. Seeto, S. Rayawa, S. Kuruyawa, A. Nasila, B. Oloni, A. Rati Ram, P. Saunivalu, P. Singh and E. Tegu 2004. Early Lapita settlement site at Bourewa, southwest Viti Levu Island, Fiji. Archaeology in Oceania 39:139-143. doi.org/10.1002/ j.1834-4453.2004.tb00571.x.

Nunn, P.D., S. Matararaba, T. Ishimura, R. Kumar and E. Nakoro 2005. Reconstructing the Lapitaera geography of northern Fiji: A newly-discovered Lapita site on Yadua Island and its implications. New Zealand Journal of Archaeology 26 (2004):41-55.

Nunn, P.D., S. Matararaba, R. Kumar, C. Pene, L. Yuen and M.R. Pastorizo 2006. Lapita on an island in the mangroves? The earliest human occupation at Qoqo Island, southwest Viti Levu, Fiji. Archaeology in New Zealand 49:205-212.

Nunn, P.D., T. Ishimura, W.R. Dickinson, K. Katayama, F. Thomas, R. Kumar, S. Matararaba, J. Davidson and T. Worthy 2007. The Lapita occupation of Naitabali, Moturiki Island, Central Fiji. Asian Perspectives 46:96-132. doi.org/10.1353/asi.2007.0009. 
O’Day, S., P. O’Day and D. Steadman 2004. Defining the Lau Context: Recent findings on Nayau, Lau Islands, Fiji. New Zealand Journal of Archaeology 25(2003): 31-56.

Palmer, B. 1966. Lapita style potsherds from Fiji. Journal of the Polynesian Society 75:373-377.

Parke, A. 2000. Coastal and inland Lapita sites in Vanua Levu, Fiji. Archaeology in Oceania 35:116-119. doi.org/10.1002/j.1834-4453.2000.tb00464.x.

Petchey, F.J. 1995. The archaeology of Kudon: The archaeological analysis of Lapita ceramics from Mulifanua, Samoa and Sigatoka, Fiji. Unpublished MA thesis, University of Auckland, Auckland.

Petchey, F., M. Spriggs, S. Bedford, F. Valentin and H.R. Buckley 2014. Radiocarbon dating of burials from the Teouma Lapita cemetery, Efate, Vanuatu. Journal of Archaeological Science 50:227-242. doi.org/10.1016/j.jas.2014.07.002.

Petchey, F., M. Spriggs, S. Bedford and F. Valentin 2015. The chronology of occupation at Teouma, Vanuatu: Use of a modified chronometric hygiene protocol and Bayesian modeling to evaluate midden remains. Journal of Archaeological Science: Reports 4:95-105. doi.org/10.1016/j.jasrep.2015.08.024.

Piroutet, M. 1917. Etude stratigraphique sur la Nouvelle-Calédonie. Imprimerie Protat frères, Mâcon.

Poulsen, J. 1987. Early Tongan prehistory: The Lapita period on Tongatapu and its relationships. Two volumes. Terra Australis 12. Department of Prehistory, RSPacS, The Australian National University, Canberra.

Sand C. 1993. Données archéologiques et géomorphologiques du site ancien d'Asipani, Futuna (Polynésie occidentale). Journal de la Société des Océanistes 96(2):117-144. doi.org/10.3406/jso.1993.2928.

Sand, C. 1994. Entre mer et montagne. Inventaire archéologique de la Commune de Païta (Province Sud). Les cahiers de l'archéologie en Nouvelle-Calédonie 4. Département Archéologie, Service des Musées et du Patrimoine de Nouvelle-Calédonie, Nouméa.

Sand, C. 1996. Le début du peuplement Austronésien de la Nouvelle-Calédonie. Les cahiers de l'archéologie en Nouvelle-Calédonie 6. Département Archéologie, Service des Musées et du Patrimoine de Nouvelle-Calédonie, Nouméa.

Sand, C. 1998a. Archaeological report on localities WKO013A and WKO013B of the site of Lapita (Koné, New Caledonia). Journal of the Polynesian Society 107(1):7-33.

Sand, C. 1998b. Recent archaeological research in the Loyalty Islands of New Caledonia. Asian Perspectives 37(2):194-223.

Sand, C. 1998c. Archaeological research on Uvea Island, Western Polynesia. New Zealand Journal of Archaeology 18(1996):91-123.

Sand, C. 1999. The beginning of Southern Melanesian Prehistory: The St Maurice-Vatcha Lapita site, New Caledonia. Journal of Field Archaeology 26(3):307-323. doi.org/10.1179/jfa.1999.26.3.307.

Sand, C. 2010. Lapita Calédonien: Archéologie d'un premier peuplement Insulaire Océanien. Collection Travaux et Documents Océanistes 2. Société des Océanistes, Paris. doi.org/10.4000/books.sdo.1128.

Sand, C. and A. Ouetcho 1992. Bwede ko-tchon tchuvan-vare kein (Des Rivières Déviées par les Ancêtres). Premier inventaire archéologique de la Commune de Yaté, Province Sud de la Nouvelle-Calédonie. Les cahiers de l'archéologie en Nouvelle-Calédonie 1. Département Archéologie, Service des Musées et du Patrimoine de Nouvelle-Calédonie, Nouméa. doi.org/10.4000/books.editionsmsh.2782.

Sand, C., F. Valentin, T. Sorovi-Vunidilo, J. Bole, A. Ouetcho, S. Matararaba, J. Naucabalavu, D. Baret and L. Lagarde 1999a. Cikobia-I-Ra, Archaeology of a Fijian Island. Les cahiers de l'archéologie en Nouvelle-Calédonie 9. Département Archéologie, Service des Musées et du Patrimoine de NouvelleCalédonie, Nouméa. doi.org/10.4000/books.editionsmsh.2782. 
Sand, C., J. Bole and A. Outecho 1999b. Fichier d'inventaire des sites archéologiques et traditionnels de la Province des Iles Loyauté: Programme 1992-1997. Unpublished. Département Archéologie du Service des Musées et du Patrimoine de Nouvelle-Calédonie, Nouméa.

Sand, C., J. Bole, A. Outecho and D. Baret 2000. Recherches archéologiques sur le site Lapita de Goro (Yaté, Province Sud). Unpublished, Département Archéologie du Service des Musées et du Patrimoine de NouvelleCalédonie, Nouméa.

Sand, C., J. Bole, A. Outecho and D. Baret 2001. Nouvelles données sur le Lapita en Province Nord (NouvelleCalédonie). Unpublished. Département Archéologie du Service des Musées et du Patrimoine de Nouvelle-Calédonie, Nouméa.

Sand, C., J. Bole, A. Outecho and D. Baret 2002. Site LP0023 of Kurin: Characteristics of a Lapita settlement in the Loyalty Islands (New Caledonia). Asian Perspectives 41:129-147. doi.org/10.1353/ asi.2002.0010.

Sand, C., I. Lilley, F. Valentin, J. Bolé, B. Gony, and D. Baret 2010. Tiga (Iles Loyauté). Préhistoire et Ethno-archéologie d'une île Mélanésienne en marge. In F. Valentin and M. Hardy (eds), Hommes, milieux et traditions dans le Pacifique Sud, pp. 33-46. De Boccard, Paris.

Sand, C., M. Terebo and L. Lagarde 2013. Le passé de Deva. Archéologie d’un Domaine Provincial Calédonien. Archeologia Pasifika 2. Institut d'archéologie de la Nouvelle-Calédonie et du Pacifique (IANCP), Nouméa.

Sémah, A.-M. and J.-C. Galipaud 1992. La fouille du site LAPITA de Patho (Ile de Maré, NouvelleCalédonie). Rapport d'Activités, Sciences Sociales, Archéologie No. 5. ORSTOM, Nouméa.

Sheppard, P.J. 2010. Into the great ocean: Lapita movement into Remote Oceania. In C. Sand and S. Bedford (eds), Lapita: Ancêtres Océaniens/Oceanic ancestors, pp. 105-117. Museé du quai Branly and Somogy, Paris.

Sheppard, P.J., S. Chiu and R. Walter 2015. Re-dating Lapita movement into Remote Oceania. Journal of Pacific Archaeology 6(1):26-36.

Skelly, R., B. David, F. Petchey and M. Leavesley 2014. Tracking ancient beach-lines inland: 2600-yearold dentate-stamped ceramics at Hopo, Vailala River region, Papua New Guinea. Antiquity 88(340):470-487. doi.org/10.1017/S0003598X00101127.

Smart, C. n.d. [1969]. Notes on the pottery sequence obtained from Southern New Caledonia. Unpublished. The Australian National University, Canberra.

Specht, J. 1968. Preliminary report of excavations on Watom Island. Journal of the Polynesian Society 77(2):117-134.

Specht, J. 1991a. Report on fieldwork in West New Britain Province, January 1991. Unpublished. Australian Museum, Sydney.

Specht, J. 1991b. Kreslo: A Lapita pottery site in southwest New Britain, Papua New Guinea. In J. Allen and C. Gosden (eds), Report of the Lapita Homeland Project, pp. 189-204. Occasional Papers in Prehistory 20. Department of Prehistory, RSPacS, The Australian National University, Canberra.

Specht, J. and C. Gosden 1997. Dating Lapita pottery in the Bismarck Archipelago, Papua New Guinea. Asian Perspectives 36(2):175-199.

Specht, J. and G.R. Summerhayes 2007. The Boduna Island (FEA) Lapita site, Papua New Guinea. In J. Specht and V. Attenbrow (eds), Archaeological studies of the middle and late Holocene, Papua New Guinea, Part II, pp. 51-103. Technical Reports of the Australian Museum 20. Australian Museum, Sydney. doi.org/10.3853/j.1835-4211.20.2007.1474. 
Specht, J. and R. Torrence 2007a. Pottery of the Talasea area, West New Britain Province, In J. Specht (ed.), Archaeological studies of the Middle and Late Holocene, Papua New Guinea, pp. 131-196. Technical Reports of the Australian Museum 20. Australian Museum, Sydney. doi.org/10.3853 /j.1835-4211.20.2007.1476.

Specht, J. and R. Torrence 2007b. Lapita all over: Land-use on the Willaumez Peninsula, Papua New Guinea. In S. Bedford, C. Sand and S.P. Connaughton (eds), Oceanic explorations: Lapita and Western Pacific settlement. pp. 71-96. Terra Australis 26. ANU E Press, Canberra. doi.org/10.22459/ TA26.2007.

Specht, J., J. Hollis and C. Pain 1981. Report on archaeological fieldwork West New Britain, Papua New Guinea. Unpublished. The Australian Museum, Sydney.

Specht, J., R. Fullagar, R. Torrence and N. Baker 1988. Prehistoric obsidian exchange in Melanesia: A perspective from the Talasea sources. Australian Archaeology 27:3-16.

Specht, J., C. Gosden, J. Webb, W. Boyd and I. Lilley 1992. Report on archaeological research in West New Britain Province, PNG January-February 1992. Unpublished. The Australian Museum, Sydney.

Spennemann, D.H.R. 1989. 'Ata 'a Tonga mo 'Ata o Tonga: Early and later prehistory of the Tongan Islands. Unpublished PhD thesis, The Australian National University, Canberra.

Spriggs, M. 1990. The changing face of Lapita: Transformation of a design. In M. Spriggs (ed.), Lapita design, form and composition: Proceedings of the Lapita Design Workshop, Canberra, December 1988, pp. 83-122. Occasional Papers in Prehistory 19. Department of Prehistory, RSPacS, The Australian National University, Canberra.

Spriggs, M. 1991. Nissan: The island in the middle. Summary report on excavations at the north end of the Solomons and south end of the Bismarcks. In J. Allen and C. Gosden (eds), Report of the Lapita Homeland Project, pp. 222-243. Occasional Papers in Prehistory 20. Department of Prehistory, RSPacS, The Australian National University, Canberra.

Summerhayes, G.R. 2000a. Lapita interaction. Terra Australis 15. Department of Archaeology and Natural History and the Centre for Archaeological Research, The Australian National University, Canberra.

Summerhayes, G.R. 2000b. Recent archaeological investigations in the Bismarck Archipelago, Anir, New Ireland Province, Papua New Guinea. Bulletin of the Indo-Pacific Prehistory Association 19:167-174.

Summerhayes, G.R. and I. Scales 2005. New Lapita pottery finds from Kolombangara, Western Solomon Islands. Archaeology in Oceania 40:14-20. doi.org/10.1002/j.1834-4453.2005.tb00575.x.

Summerhayes, G.R., L. Matisoo-Smith, H. Mandui, J. Allen, J. Specht, N. Hogg and S. McPherson 2010. Tamuarawai (EQS): An Early Lapita site on Emirau, New Ireland, PNG. The Journal of Pacific Archaeology 1:62-75.

Swadling, P. (ed.) 1992. Places of cultural and natural heritage significance in West New Britain: A report for West New Britain Provincial Tourist Bureau. Unpublished. PNG National Museum, Boroko.

Swadling, P., B. Hauser Schäublin, P. Gorecki and F. Tiesler 1988. The Sepik-Ramu: An introduction. PNG National Museum, Boroko.

Terrell, J. and R. Welsch 1997. Lapita and the temporal geography of prehistory. Antiquity 71:548-572. doi.org/10.1017/S0003598X0008532X.

Tochilin, C., W.R. Dickinson, M.W. Felgate, M. Pecha, P. Sheppard, F.H. Damon, S. Bickler and G.E. Gehrels 2012. Sourcing temper sands in ancient ceramics with $\mathrm{U}-\mathrm{Pb}$ ages of detrital zircons: A Southwest Pacific test case. Journal of Archaeological Science 39:2583-2591. doi.org/10.1016/ j.jas.2012.04.002. 
Torrence, R. and C. Stevenson 2000. Beyond the beach: Changing Lapita landscapes on Garua Island, Papua New Guinea. In A. Anderson and T. Murray (eds), Australian archaeologist: Collected papers in honour of Jim Allen, pp. 324-345. Coombs Academic Publishing, The Australian National University, Canberra.

Torrence, R., J. Specht and B. Boyd 1999. Archaeological fieldwork on Numundo and Garu Plantations, West New Britain, PNG. Report submitted to the West New Britain Provincial Government and the National Museum and Art Gallery, Papua New Guinea. Unpublished. Australian Museum, Sydney.

White, J.P. 1992. New Ireland and Lapita. In J.-C. Galipaud (ed.), Poterie Lapita et peuplement: Actes du Colloque Lapita, Nouméa, Janvier 1992, pp. 83-90. ORSTOM, Nouméa.

White, J.P. 1996. Archaeological survey in southern New Ireland. Journal de la Société des Océanistes 105: 141-146. doi.org/10.3406/jso.1997.2023.

White, J.P. 2007. Ceramic sites on the Duke of York Islands. In J. Specht (ed.), Archaeological studies of the Middle and Late Holocene, Papua New Guinea, pp. 3-50. Technical Reports of the Australian Museum No. 20, Sydney. doi.org/10.3853/j.1835-4211.20.2007.1473.

White, J.P. and J. Downie 1980. Excavations at Lesu, New Ireland. Asian Perspectives 23:193-220.

White, J.P. and M.N. Harris 1997. Changing sources: Early Lapita period obsidian in the Bismarck Archipelago. Archaeology in Oceania 32:97-107. doi.org/10.1002/j.1834-4453.1997.tb00375.x.

White, J.P. and C.V. Murray-Wallace 1996. Site ENX (Fissoa) and the incised and applied pottery tradition in New Ireland, Papua New Guinea. Man and Culture in Oceania 12:31-46.

Wickler, S. 2001. The prehistory of Buka: A stepping stone island in the Northern Solomons. Terra Australis 16. Department of Archaeology and Natural History and the Centre for Archaeological Research, The Australian National University, Canberra. 
This text is taken from Debating Lapita: Distribution, Chronology, Society and Subsistence, edited by Stuart Bedford and Matthew Spriggs, published 2019 by ANU Press,

The Australian National University, Canberra, Australia.

doi.org/10.22459/TA52.2019.01 